Themenheft Nr. 46: Eltern - Pädagog*innen - Medienkompetenzen.

Eltern und Pädagog*innen zwischen Aneignung und Vermittlung von Medienkompetenz. Herausgegeben von Thorsten Naab, Alexandra Langmeyer, Ruth Wendt und Jessica Kühn

\title{
Einstellungen und Bewertungen von Eltern an reformpädagogischen Schulen zur medienerzieherischen Praxis
}

\section{Ergebnisse anhand innovativer Abfragedimensionen der quantitativ- explorativen MünDig-Studie}

\author{
Julia Kernbach ${ }^{1}$ (D), Paula Bleckmann ${ }^{1}$ (D), Benjamin Streit ${ }^{1}$ (D) und Brigitte Pemberger ${ }^{1}$ (D) \\ ${ }^{1}$ Alanus Hochschule für Kunst und Gesellschaft
}

\begin{abstract}
Zusammenfassung
Bisher sind Eltern als zentrale Akteure gelingender Medienbildung nach ihrem medienerzieherischen Handeln in der Familie, aber kaum nach einer differenzierten Bewertung der schulischen Praxis befragt worden. Für die MünDig-Studie, eine bundesweit im Herbst 2019 durchgeführte Onlinebefragung von Eltern, älteren Schülerinnen und Schülern und Lehrkräften an reformpädagogischen Schulen, erwiesen sich gängige Befragungsinstrumente als ungeeignet. Daher wurde ein multidimensionales Befragungsinstrument entwickelt, das medienerzieherische Einstellungen und Aktivitäten differenziert nach verschiedenen Medien (mit und ohne Bildschirm), dem Alter des Kindes sowie Lern-/Bildungszielen (Medienkompetenzrahmen NRW plus 4 zusätzliche Bereiche) erfasst. Die in der MünDig-Studie befragten Montessori- und Waldorfeltern bewerten die schulische medienerzieherische Praxis mehrheitlich positiv, sehen jedoch in Teilbereichen Defizite. Zufriedenheit zeigt sich bezüglich des pädagogischen Ansatzes, der «ein selbständiges, kritisches Denken und Handeln unterstützt» (88\%) und der Förderung von Medienmündigkeit durch den Einsatz von Medien ohne Bildschirm (88\%). Für die oberen Klassen wird mehr Einsatz digitaler Bildschirmmedien gewünscht (Eltern-Zufriedenheit $70 \%$ in den Klassen 1-3, $44 \%$ in den Klassen 10-13), ebenso deutlich mehr pädagogische wie technische Unterstützung. Zufriedenheit besteht über Aktivitäten der Schule, die Kinder und Jugendliche im sozialen Miteinander (74 \%) zum Schutz vor Digital-Risiken im realen Leben stärken. Erstmals ermöglichen die entwickelten innovativen Bewertungsdimensionen die Beschreibung einer interessanten medienerzieherischen Haltung, die jenseits einer angenommenen Dichotomie «bewahrpädagogische» wie auch «aktiv-handlungsorientierte» Ansätze zu vereinen scheint.
\end{abstract}


Parental Views on Media Education Practice at Montessori and Steiner Schools. Results of the Quantitative Explorative MünDig Study Using an Innovative Multidimensional Survey Instrument

\begin{abstract}
Beliefs and ratings of parents at Montessori and Waldorf Schools on the practice of media education - results from the quantitative-explorative MünDig Study by using innovative assessment dimensions Parents as central actors in the field of media education have been studied regarding "parental mediation» of media use in the home, but rarely regarding their opinion on media education practice at their school. Available survey instruments for doing this turn out to fit poorly with the target group of the presented MünDig Study. Therefore, a new survey tool was developed, which was conducted in Germany as a nationwide online survey in fall 2019. The tool differentiates between the type of medium, different ages of the child and between different educational/learning goals. The responding parents' opinion of media education practice at school is positive on the whole, but they see deficits in defined areas. Parents are content the way school fosters media maturity through a "pedagogy which fosters self-determined and critical thoughts and actions» (88\%), as well as through the use of non-screen media in class (88\%). They would favor more use of digital screen media in higher classes (Class 1-3 $70 \%$ content, Class 10-13 only $44 \%$ content). The innovative tool makes it possible to describe an interesting frame of mind regarding media education. It seems to lie the often-assumed dichotomy between «restrictive/protectionist» and "active-action-oriented» approaches to media education by encompassing both.
\end{abstract}

\title{
1. Herausforderungen für die Untersuchung von Medienerziehung ${ }^{1}$ im reformpädagogischen ${ }^{2}$ Setting
}

Nicht erst seit Beginn der Ausbreitung der SARS-CoV-2-Pandemie berichten der Bund der Freien Waldorfschulen wie auch die verschiedenen Montessori-Verbände und -Weiterbildungsorganisationen ${ }^{3}$ von einer zunehmenden Nachfrage nach medienpädagogischen Weiterbildungen, Workshops und Vorträgen. Obwohl die Integration

1 Das Forschungsprojekt, in dessen Kontext die MünDig-Studie durchgeführt wurde, trägt den Titel «Medienerziehung an reformpädagogischen Bildungseinrichtungen», weswegen im Beitrag meist der Begriff Medienerziehung verwendet wird. Aufgrund seiner umfassenderen und somit besser zum abgedeckten Themenfeld in der Befragung passenden Bedeutung wird von den Autorinnen und Autoren mittlerweile der Begriff Medienbildung nach Marotzki und Jörissen (2008) zur Beschreibung des Untersuchungsgegenstandes als treffender angesehen.

2 Obgleich eine Vielzahl weiterer nennenswerter reformpädagogischer Strömungen existieren, sind hier soweit nicht anders expliziert, mit Reformpädagogik Waldorf-und Montessori-Pädagogik gemeint, da sie den weit überwiegenden Teil der Befragungsteilnehmenden der MünDig-Studie darstellen.

3 Montessori-Dachverband Deutschland (MDD), Deutsche Montessori-Vereinigung (DMV), Deutsche Montessori-Gesellschaft (DMG). 
von medienpädagogischen Themen in bestehende Montessori-Diplomkurse sowie Waldorf-Ausbildungslehrgänge deutlich geringer als in der staatlichen Lehramtsausbildung ausfällt, lässt sich eine Zunahme der Implementierung von Modulen dieses Themenkomplexes konstatieren.

Die jeweiligen Verbände unterhalten eigene bundesweite Arbeitsgruppen zum Thema Medienerziehung. Waldorf-Ehemalige (jüngste Teilstichprobe $N=1.536$ bei der Waldorf-Ehemaligenbefragung (Graudenz 2021)) nannten bei der Frage nach den wichtigsten Herausforderungen, mit denen sie Waldorfschulen heute konfrontiert sehen, mit $33 \%$ am häufigsten den Themenkomplex «Neue Medien/Digitalisierung». Zeitgleich mit diesem teilweise «schlechten» Urteil in Bezug auf die Medienbildung verzeichnen Waldorf- und Montessori-Schulen in Deutschland stetigen Zulauf, zumal heute 2 von 100 eingeschulten Kindern eine Schule mit solch pädagogischer Orientierung besuchen. ${ }^{4}$ Liegt darin ein Widerspruch?

Livingstone und Kollegen berichten, dass Eltern, die sich selbst als hochgradig "digital kompetent» einschätzen, für ihre Kinder ein spätes Einstiegsalter für das Treffen eigener Entscheidungen in virtuellen Welten wünschen (Livingstone, BlumRoss, und Dongmiao 2018), was das private Medienerziehungsverhalten von IT-Milliardären wie Bill Gates (Microsoft), Jeff Bezos (Amazon) oder Steve Jobs (Apple) erklären könnte. Den jeweils eigenen Kindern stellten diese die ersten Smartphones bzw. Tablets frühestens ab 14 Jahren zur Verfügung (Bleckmann und Nartschenko 2019). Hiervon scheint eine inspirierende Dynamik auszugehen, aufgrund derer sich in den USA die «wait until $8^{\text {th }}$ ( («Warte bis Klasse 8») Bewegung formierte (www.waituntil8th. org), der sich bereits Tausende von Eltern angeschlossen haben. Das deutsche Äquivalent nennt sich «Smarter Start ab 14» (www.smarterstartab14.de). Das Vorliegen von Hinweisen auf die o. g. medienerzieherischen Schwächen wie auch Stärken (vgl. Abschnitt 3.2) macht das Thema «Medienerziehung und Reformpädagogik» zu einem interessanten Forschungsfeld. Die hohe Relevanz der Zusammenarbeit von pädagogischen Fachkräften und Eltern in reformpädagogischen Lernkulturen (vgl. Abschnitt 3.1), in denen Eltern als zentrale Akteure gelingender Bildungsprozesse im Allgemeinen und daher auch der Medienbildung betrachtet werden, lässt speziell auch die Untersuchung von Eltern-Einstellungen und -Bewertungen wichtig erscheinen. Darüber wird im vorliegenden Beitrag berichtet, indem im ersten Teil einerseits die sich daraus ergebenden Herausforderungen in Schule und Elternhaus beschrieben und andererseits aus der Forschung entwickelte mögliche Lösungsansätze für diese Zielgruppe dargelegt werden. Der zweite Teil fokussiert die Beschreibung des entwickelten Befragungsinstrumentes mit den innovativen Bewertungsdimensionen, das

4 Allein an den über 200 Waldorfschulen in Deutschland wurden im Jahr 2019 rund 85500 Schülerinnen und Schüler beschult, dazu kommen die Schülerinnen und Schüler an den etwa 400 Montessori-Schulen, von denen etwa 300 Grundschulen sind. Somit besuchen Schülerinnen und Schüler aus über 100000 Elternhäusern eine reformpädagogisch orientierte Schule (Statistisches Bundesamt 2019). 
zu den empirischen Forschungsergebnissen und der erstmaligen Beschreibung einer medienerzieherischen Haltung führt, die «bewahrpädagogische» wie auch «aktivhandlungsorientierte» Ansätze zu vereinen scheint.

\subsection{Erste Herausforderung: Elternrolle bisher nicht «auf Augenhöhe» untersucht}

Die meisten Befragungsinstrumente zum Thema «Eltern und Medienerziehung» zielen auf die Erfassung der Regulierung der kindlichen Nutzung von Bildschirmmedien durch die Eltern im Elternhaus ab. Historisch wurde dies zuerst für das Fernsehen, sukzessive dann auch für weitere neuere Medien erforscht. Es führte zu folgender, mit leichter Veränderung auf alle Bildschirmmedien bezogenen, Einteilung elterlicher Medienerziehungsstile (Valkenburg et al. 1999; Böcking 2006; Nathanson 1999):

- restriktive elterliche Begleitung (restrictive mediation),

- aktive beziehungsweise instruktive elterliche Begleitung (active/instructive mediation),

- (Social) Co-Viewing bzw. Laissez-Faire.

Aktuelle Studienergebnisse zeigen, dass gerade bei jüngeren Kindern die restriktive elterliche Begleitung mit einer geringeren Prävalenz problematischer Nutzungsformen korreliert (Livingstone, Blum-Ross, und Dongmiao 2018). Im Vergleich zu den oben genannten Medienerziehungsstilen hat jedoch laut Ergebnissen des «Berliner Längsschnitt Medien» (Mößle und Bleckmann 2015) die Nicht-Verfügbarkeit eigener Mediengeräte in Kinderhand eine noch stärkere protektive Wirkung in Bezug auf alle drei möglichen Problemdimensionen: Zeit (ausufernde Bildschirmmediennutzung), Inhalt (z. B. nicht-altersgerechte Inhalte) und Funktion (z. B. suchtartige Nutzung). Befragungsinstrumente für die Zielgruppe Eltern differenzieren im Bereich der «aktiven Begleitung» nicht nach verschiedenen Kompetenzbereichen der Medienbildung, sondern fragen Items ab wie «Ich interessiere mich dafür, was mein Kind im Internet macht» oder «lch spreche mit meinem Kind über seine Medienerlebnisse». Somit ist die Elternbefragung weitaus undifferenzierter als etwa die Abfrage medienerzieherischer Einstellungen und Praxis von Pädagoginnen und Pädagogen in der Tradition der «Technologie-Akzeptanz» (TAM)-Modellierung, der Belief-Forschung und weiteren Forschungstraditionen (für einen Überblick siehe Schmidt 2020). Eltern wird damit u.E. eine grosse Verantwortung zur Regulierung der kindlichen Mediennutzung, zugleich aber implizit eine eingeschränkte bzw. untergeordnete Deutungsmacht in der Medienerziehung zugeschrieben. Pädagogische Fachkräfte sind aufgefordert, im Kontext Schule neben der direkten Förderung von Medienkompetenz der Kinder und Jugendlichen im Klassenzimmer, Eltern zur Steuerung der häuslichen Mediensozialisation zu beraten und zu begleiten. Umgekehrt jedoch werden Eltern höchst selten 
als an Medienbildungsprozessen beteiligte Akteure zur Mediensozialisation in der Schule eingebunden. Im Rahmen des Auf- bzw. Ausbaus einer Erziehungspartnerschaft «auf Augenhöhe» (vgl. auch Abschnitt 3.2) könnte hingegen letzteres ebenfalls von Bedeutung sein.

Darüber, wie Eltern die Medienerziehung an der Schule ihrer Kinder bewerten, finden sich bislang kaum Forschungsergebnisse (siehe Abschnitt 3.3), sofern doch untersucht, wird dabei eine Differenzierung nach unterschiedlichen Lernzielen und Altersstufen vernachlässigt.

\subsection{Zweite Herausforderung: Ungenügende Passung zwischen gängigen Befragungsinstrumenten und reformpädagogischen Lehrkräften und Eltern}

Die zweite Herausforderung bezieht sich nicht spezifisch auf Eltern, sondern auf reformpädagogisch orientierte Eltern und Lehrkräfte. Um die Eignung bestehender Befragungsinstrumente für diese Gruppe zu überprüfen, wurde erstens eine Analyse bisher verwendeter Instrumente der letzten 10 Jahre erbracht und zweitens eine qualitative Vorstudie mit Lehrkräften und Eltern durchgeführt. Zu den untersuchten Befragungsinstrumenten gehören beispielsweise «Bertelsmann Monitor Digitale Bildung» (Thom et al. 2018), «DigCompEdu» (Redecker 2017) und «Länderindikator Digital» (Lorenz et al. 2014), aber auch die o. g. Einstellungs- und Beliefs-Modelle (Schmidt 2020). Diese sind zwar mit gängigen schulischen Vorgaben zur Bildung in der digitalen Welt (LVR Zentrum für Medien und Bildung; Kultusministerkonferenz 2016) kompatibel, jedoch erwies sich deren Passungsgrad im Rahmen der qualitativen Vorstudie für die zu untersuchende Befragungsgruppe aus folgenden Gründen insgesamt als zu gering:

1. Mangelnde Differenzierung nach Entwicklungsphasen oder zumindest Altersgruppen der Kinder und Jugendlichen.

2. Unzureichende Differenzierung zwischen medienpädagogischen Aktivitäten mit digitalen Bildschirmmedien (mit Bildschirm) und mit «analogen» Medien (ohne Bildschirm).

3. Mangelnde Einbeziehung mehrerer in der Vorstudie als wichtig benannter Bereiche wie beispielsweise die der ressourcenorientierten Prävention von Digital-Risiken, der Verarbeitung problematischer Medienerlebnisse und übergreifend auch der medienpädagogischen Elternzusammenarbeit.

4. Ambivalent zeigen sich eine mangelnde Differenzierung nach Lernziel und ein daraus (teilweise) hervorgerufener Widerstand gegen das Zerstückeln «ganzheitlicher» Bildungsprozesse in einzelne Kompetenzbereiche. Dies stimmt mit Befunden überein, nach denen insbesondere Waldorf-Lehrkräften das Denken in voneinander getrennten Kompetenzbereichen im Zusammenhang mit Lehr- und Lernprozessen tendenziell wenig geläufig ist (Richter 2006/2016; Frielingsdorf 2019). 
5. Auch die Forderung nach einer Altersdifferenzierung erscheint plausibel, da «die Idee der Eigengesetzlichkeit der kindlichen Entwicklung» (Skiera 2010, 219) und die darauf basierenden, ausgearbeiteten Entwicklungslehren sowohl in der Montessori-, als auch in der Waldorfpädagogik eine zentrale Rolle spielt.

\section{Lösungsansätze: Altersspezifisch, lernzielspezifisch und medienspezifisch befragen}

Diese Feststellungen führten gemeinsam mit einer Berücksichtigung empirischer wie theoretischer Befunde, auf welche wir im Abschnitt 3.3 näher eingehen, bei der Entwicklung des innovativen Befragungsinstrumentes zu den folgenden Entscheidungen: Erstens, allen drei Gruppen von Befragten (Eltern, Lehrkräften und älteren Schülerinnen und Schülern) wird ein in Teilen sehr ähnlicher oder gleicher Fragebogen vorgelegt. Zweitens, die sechs Kompetenzbereiche des Medienkompetenzrahmen NRW (für eine Auflistung vgl. Abschnitt 5.1) werden übernommen. Für jeden dieser Bereiche werden drittens je gleich viele Aktivitäten mit und ohne Einsatz von Bildschirmmedien in das Befragungsinstrument aufgenommen. Dies scheint mit dem erweiterten Verständnis von Medienerziehung um Aspekte der Zuhilfenahme nichtdigitaler Medien in der Tradition von Baacke (1997) gut kompatibel zu sein. Die hohe Bedeutung von Sprachförderung für die Förderung von Medienkompetenz lässt sich in mehreren Hinweisen bei ihm finden. Auch Irion fasst die Medienkompetenzbereiche von NRW als nicht direkt auf spezifische Techniken und Strategien der Bedienung von digitalen Technologien ausgerichtete, sondern auf Handlungsprozesse in Bildungs- und Lebenssituationen in der digital geprägten und gestaltbaren Welt abzielende Kompetenzen zusammen (Irion 2020, 67).

Viertens werden die Bereiche «Elternzusammenarbeit», «Verarbeitung belastender Medienerlebnisse» (J. Zimmer und K. M. Zimmer 2020) sowie «Ressourcenorientierte (Mediensucht-)Prävention» (Bleckmann und Mößle 2014; Wiesmann 2019) hinzugefügt. Fünftens wird für jede Beispielaktivität erfragt, in welchem Alter diese als sinnvoll erachtet bzw. auch praktiziert wird.

\section{Forschungsbefunde im Dreieck Medienerziehung, Reformpädagogik und Elternzusammenarbeit}

\subsection{Elternzusammenarbeit im reformpädagogischen Kontext}

Auf Grundlage der Konzeptionen von Rudolf Steiner und Maria Montessori (vgl. Fussnote 3) wird eine funktionierende Erziehungspartnerschaft angestrebt, deren Beteiligte sich für gemeinsame pädagogische Zielsetzungen interessieren und sich um 
einen dialogischen Austausch bemühen. Dabei betont Dietz (2011, 287), es liege in der Natur hochwertiger Kooperation, dass diese unter Zielsetzungen betrieben werde, die nicht anfangs definiert und dann eins zu eins «verwirklicht» werden könne. Was Dietz für den Kontext Waldorf-Pädagogik beschreibt, weist deutliche Übereinstimmungen mit der in der Literatur beschriebenen Grundlage der zeitgenössischen Montessori-Pädagogik auf (Onken 2017; Stein 2017; Werner-Andrews 2017). Gleichzeitig halten Peitz, Harring und Müller $(2017,333)$ kritisch fest, im reformpädagogischen Kontext erscheine insbesondere an Waldorfschulen die elterliche Einbeziehung bisweilen als simulierte Partizipation, da in Bezug auf Konzeptentwicklungen fast ausschliesslich die professionellen Pädagoginnen und Pädagogen als die zentralen und einflussreichen Entscheidungsträger fungierten.

\subsubsection{Aspekte der Elternrolle im reformpädagogischen Kontext und Konsequenzen für das Studiendesign}

Die Zusammenarbeit von Schule und Elternhaus charakterisiert Dietz als im Wesentlichen geprägt durch Schulgründungen, die meist durch Elterninitiative und andererseits durch Selbstverwaltung der Bildungseinrichtungen getragen werden. Dies erfordere von Anbeginn eine intensive Zusammenarbeit zwischen Eltern und Schule bzw. Lehrkräften (Dietz 2011, 283). Peitz und Kollegen konstatieren allerdings im Hinblick auf die Elternpartizipation eine gewisse Paradoxie, da sich Eltern mit der Wahl einer Reformschule zwar für eine erhöhte Beteiligung an schulischen Aktivitäten entscheiden würden (Peitz, Harring, und Müller 2017), der Grad an Offenheit und das Ausmass der tatsächlichen Mitbestimmung der Eltern stelle sich jedoch in der praktischen Handhabung selbst bei einrichtungsspezifischer konzeptioneller Verankerung als unterschiedlich dar. Gleichwohl könne dies als partnerschaftliches Verhältnis beschrieben werden (vgl.Peitz, Harring, und Müller 2017, 327). Im Unterschied zur Praxis an staatlichen Regelschulen werden Eltern der meisten Schulen in privater, selbstverwalteter (freier) Trägerschaft mit Schuleintritt ihrer Kinder Mitglied des Trägervereins und dadurch selbst zum Träger der von ihnen explizit gewählten Schule. Nebst ihrer Mitwirkung im Sozialen der Schulkultur leisten sie einen monatlich festgelegten, meist einkommensabhängigen Schulkostenanteil. Das Passungsverhältnis Eltern-Schule im reformpädagogischen Kontext kann somit als ein besonderes angesehen werden, sollte aber nicht vorschnell der Überbewertung unterliegen: Erstens befinden sich nicht alle reformpädagogischen Schulen in privater Trägerschaft, zweitens fällt die Handhabung in der Praxis unterschiedlich aus und drittens sind trotz des offenbar besonderen Passungsverhältnisses Schulwechsel keine Seltenheit.

In Bezug auf die Elternzusammenarbeit wird sowohl an Montessori- wie auch an Waldorfschulen eine Ausgestaltung befürwortet, welche «die klassischen asymmetrischen Muster in der Beziehung zwischen Eltern und Fachkräften hinter sich lässt» (Stange 2013, 30), was sich überdies mit den aktuell geforderten Entwicklungen im 
staatlichen Bildungssystem (insbesondere in der Frühpädagogik) deckt. An diesem Ideal orientieren wir die Entscheidung, in der MünDig-Studie die Eltern in weiten Teilen mit demselben innovativen multidimensionalen Instrument zu befragen wie die pädagogischen Fachkräfte.

\section{2 «Alternative» Formen medienerzieherischer Praxis in der Reformpädagogik und darüber hinaus}

Historisch gesehen sind reformpädagogische Ansätze jeweils als Ansätze einer «neuen» Schul- und Lernkultur zu verstehen, die sich nie als eine homogene Bewegung darstellen, sondern sich als eine Vielfalt darunter zusammengefasster Ansätze und Perspektiven begreifen lassen (Wolf 2017, 339). Wolf sieht in reformpädagogischen Ansätzen, insbesondere der Montessori-Pädagogik mit dem Grundgedanken, dass jedes einzelne Kind «am Material» seine Lernerfahrungen sammeln darf, eine Prädisposition, sich prinzipiell rasch auf den Einsatz digitaler Medien in Bildungsprozessen einzulassen. Die Frage nach einer empfehlenswerten medienerzieherischen Praxis wird hingegen durchaus unterschiedlich beantwortet, was sich auf die jeweils zugrundeliegende Anthropologie und dem sich daraus abgeleiteten entsprechenden Bildungs- und Erziehungsverständnis zurückführen lässt. Während über das Ziel medienerzieherischer Praxis weitgehend Einigkeit herrscht, scheiden sich die Geister an der Frage nach der methodisch-didaktischen Gestaltung der «Wege» hin zu diesem Ziel. Für die Benennung des Ziels erfährt in der Reformpädagogik der Begriff «Medienmündigkeit» breite Akzeptanz (Bleckmann 2020; Kullack-Ublick und Arbeitskreis Medienmündigkeit und Waldorfpädagogik im Bund der Freien Waldorfschulen 2015). Bleckmann postuliert, die Aufgabe von Bildung auch und gerade im «digitalen Zeitalter» bestehe darin, Kinder auf dem Weg zu einem gemeinschaftsfähigen und selbständig denkenden Erwachsenen zu begleiten. Dieser Erwachsene sollte sich Fähigkeiten zur dosierten, aktiven, kreativen und technisch versierten Handhabung digitaler Medien erworben haben und selbstbestimmt darüber entscheiden können, welchen Anteil seiner Lebenszeit er mit Bildschirmmedien verbringt (Bleckmann 2020). Die Abweichung von umfassenden Medienkompetenz-Definitionen wie der von Hüther und Schorb ist u. E. gering:

«Medienkompetenz ist die Fähigkeit auf der Basis strukturierten zusammenschauenden Wissens und einer ethisch fundierten Bewertung der medialen Erscheinungsformen und Inhalte, sich Medien anzueignen, mit ihnen kritisch, genussvoll und reflexiv umzugehen und sie nach eigenen inhaltlichen und ästhetischen Vorstellungen, in sozialer Verantwortung sowie in kreativem und kollektivem Handeln zu gestalten.» (Hüther und Schorb 2005, 262). 
Aus der Perspektive der Anthropologie der Waldorfpädagogik empfiehlt Hübner hierfür einerseits, den Kindern alles zu bieten, was ihrer individuellen und persönlichen Reifeentwicklung förderlich sei und anderseits, das von ihnen fernzuhalten, dem sie noch nicht gewachsen seien. Daraus wird basierend auf Annahmen über die zentrale Bedeutung der Sinnesentwicklung folgende konkrete Empfehlung abgeleitet:

«Zuerst müssen Kinder die reale Welt erfahren und erleben, dann können die Kinder analoge Techniken verstehen und beherrschen lernen und zuletzt, aufbauend auf diesen beiden Voraussetzungen, kann ein gründliches Verständnis der digitalen Welt erworben werden.» (Hübner 2020, 251 ff).

Aus der Perspektive der Montessori-Pädagogik bezeichnen Herbst und Bergmayr Bildschirmmedien in der formativen Phase der Kindheit als ungeeignet, da diese sich zwischen das Kind und die Welt schieben und soziale Beziehungen, den sprachlichen Austausch sowie die Bewegungs- und Sinneserfahrungen einschränken würden (Herbst und Bergmayr 2020). Dadurch würde ferner die aktive, vollleibliche und selbsttätige spielerische Verarbeitung der Wirklichkeit gestört und die Selbstaufbautätigkeit des Kindes manipuliert werden. Entwicklungsphasenspezifische, analoge Medien einbeziehende Ansätze werden in jüngerer Zeit auch ausserhalb reformpädagogischer Autorenschaft aufgegriffen (Brinda et al. 2019; Hromkovič und Lacher 2019; Hauser et al. 2020). Te Wildt empfiehlt, Heranwachsende sollten die Möglichkeit bekommen, die mediale Evolution in der Schule nachzuschreiten (Te Wildt 2015, 309).

Hingegen seien im ausserschulischen Lernfeld nach Grobbin die Eltern die wichtigsten Bildungsakteure (Grobbin 2016, 34f). Neben vielen anderen Herausforderungen obliege ihnen die Aufgabe, ihr eigenes Mediennutzungsverhalten in der Gegenwart der Kinder zumindest so zu steuern, dass Kinder die Möglichkeit bekämen, sich mit der gegenständlich-dinglichen Welt auseinanderzusetzen (Penert und Pemberger 2020).

\subsection{Schulische Medienerziehung aus Elternsicht: Empirische Befunde}

In der deutschsprachigen wie auch in der internationalen Forschungsliteratur finden sich bis auf wenige Ausnahmen (Lee 2003) kaum Studien, die die elterliche Bewertung schulischer Medienbildungspraxis detailliert untersuchen. Dies überrascht insofern, als in vielen Handreichungen für medienpädagogische Elternberatung darauf eingegangen wird, wie einer ablehnenden Haltung von Eltern gegenüber dem Einsatz von digitalen Medien in Bildungseinrichtungen (insbesondere in Kindergärten) entgegengewirkt werden könnte. Als Teilbereich einer Studie zu «Beratungs-, Handlungs- und Regulierungsbedarf aus Elternperspektive» erfasst Grobbin (2016) 
die elterliche Bewertung der Medienbildung an Kindergärten und Schulen. Demnach sehen Eltern Bildungseinrichtungen in der Pflicht, Medienbildung zu vermitteln, da sie ihre Kinder gut vorbereitet wissen möchten auf ein Leben in der «digitalen Welt». Kindergarteneltern befürworten jedoch weit überwiegend nicht die Idee, ihr Kind solle in der Bildungseinrichtung «die neuesten Medien, z. B. Tablet, kennenlernen» (Kindergarteneltern: $63 \%$ «stimme überhaupt nicht zu») bzw. «über die Gefahren des Internets aufgeklärt werden» (55\% «stimme überhaupt nicht zu»). Bei Schuleltern ist die Zustimmung zwar deutlich höher, aber keineswegs einhellig. Überraschend zeigt der Befund bei Grobbin, dass es «keine Rolle [spielt], ob Eltern von Grundschülern oder Sekundarstufenschülern gefragt werden» (Grobbin 2016, 18ff). Hingegen geben in der KIM-Studie $46 \%$ der befragten Eltern $(\mathrm{N}=1.231)$ an, einen/ein PC/Laptop/Tablet für den Schulerfolg ihrer 6- bis 13-jährigen Kinder für wichtig zu erachten (Feierabend, Plankenhorn, und Rathgeb 2019).

Zwei Befragungen von Waldorf-Schuleltern lassen sich anführen, die auch Items zur Bewertung der medienerzieherischen Praxis enthalten (für die Schweiz Brodbeck 2020; für Deutschland Barz 2019). Im Vergleich zu Eltern anderer Schulformen befürworten Waldorf-Schuleltern demnach einen deutlich späteren Digitalmedieneinsatz, der allerdings mit einem einzigen, wenig differenzierten Item abgefragt wurde: «Ab welchem Alter gehören Computer und Tablets in den Unterricht?». Während etwa ein Fünftel der Eltern an staatlichen Regelschulen die Kategorie «im Verlauf der Klassen 1 bis 4» wählte, sind es bei den Waldorf-Schuleltern ( $N=97) 0 \%$, dagegen befürworten $16 \%$ «im Verlauf der Klassen 7 bis 9 » und die übrigen Eltern die Kategorie «noch später [als Klasse 7]» (Tetzlaff und Bleckmann 2019).

\subsection{Medienerziehung ohne Bildschirm in der Praxis}

Einer Reihe von Ansätzen, die (nicht nur) in der reformpädagogischen Praxis umgesetzt werden, liegt zusammengefasst das Konzept «wenn immer möglich zuerst analog begreifen und danach ins Digitale übertragen» zugrunde. Hübner sieht für eine entwicklungsorientierte Medienpädagogik (Hübner 2020) beispielsweise Schreiben lernen mit Stift und Papier als Erlernen einer Kulturtechnik an, die zu einer analogen Medienkompetenz führt (Schreiben können mit Stift und Papier), worauf eine spätere auf digitale Medien und Techniken übertragene Medienkompetenz fusse, z. B. eine Geburtstagseinladung via E-Mail schreiben und versenden oder empfangen und verstehen können. Ebenso erfolgt in den unteren Schuljahren, insbesondere zur Unterstützung effizienter Wortschatzwiederholung in Fremdsprachen der Aufbau einer (analogen) strukturierten Wissensdatenbank mittels eines Karteikastens (DatenVerwaltung und -Strukturierung). Hier wird in den genannten Beispielen also nicht notwendigerweise Neues entwickelt und umgesetzt, sondern oftmals eine bereits 
bestehende Unterrichtspraxis auch als Medienerziehung bzw. als informatische Bildung neu deklariert. Ebenso finden sich Beispiele von Neukonzeptionen oder Weiterentwicklung: Lochkarten kommen für ein erstes Erleben und intuitives Verstehen der Binärcodierung (und paralleler binärer Suchalgorithmen) zum Einsatz, u. a. in Verbindung mit einer altersgemässen Förderung von Computational Thinking ${ }^{5}$. Ebenfalls sollen gestalterische Fähigkeiten und Grundlagen szenographischer Gestaltung erworben werden können, anhand des Schattentheaterspiels oder im Rahmen einer weitestgehend bildschirmfreien Unterrichtseinheit «Vom Standbild zum Bewegtbild»: Dabei wird zunächst ein Daumenkino gestaltet, dann ein Stop-MotionKnet-Trickfilm-Projekt umgesetzt, um daran zu erfahren, wie sich anhand von schnell nacheinander ablaufenden Einzelbildern Bewegtbilder erzeugen lassen.

Aktuell liegen noch wenige Publikationen vor, die Unterrichtseinheiten im Sinne einer «Medienerziehung (weitestgehend) ohne Bildschirm» detailliert beschreiben, und noch weniger, in denen beabsichtigte Kompetenzgewinne empirisch überprüft werden. Ausnahmen bilden z. B. www.csunplugged.org (siehe oben), für den Primarbereich die Lehrmittel «Einfach Informatik» (Hromkovič und Lacher 2019; Hauser et al. 2020) und das analoge soziale Netzwerk für die handlungs- und ressourcenorientierte Prävention von Cyber-Risiken (Sümmchen 2019), für welches immerhin eine eher knappe Beschreibung mit photographischer Dokumentation vorliegt. In einem in Vergleichsgruppen angelegten Forschungsprojekt untersucht (Penert 2019) mittels standardisiertem Testverfahren die Fähigkeiten von Waldorf-Mittelstufenschülerinnen und -schüler (Primar- und Sekundarstufe) im Bereich Algorithmen. Die quantitative Studie $(N=119)$ zeigt, dass im Computational Thinking-Test die Ergebnisse der Gruppe mit Bewegungsintervention (Treatment) signifikant besser ausfallen als die Testergebnisse der Gruppe ohne Bewegungsintervention.

Die oben exemplarisch genannten Praxisbeispiele zielen bei den Lernenden auf das Ermöglichen von Erkenntnisgewinn auf Grundlage von eigenem Erfahren und Erleben (Antonovsky 1997) ab, das u. a. mit dem an Phänomenen orientierten Ansatz reformpädagogischer Unterrichtsgestaltung viel gemeinsam hat. Zusammenfassend lässt sich konstatieren: Es findet aus der Lernenden-Perspektive in einem ersten Schritt eine basale Prinzipien erfahrende Tätigkeit auf der enaktiven und bildhaften Ebene statt, die in einem zweiten Schritt in eine symbolische und deutlich abstraktere Ebene überführt und/oder um diese ergänzt werden kann (Schwill 1993). In Anlehnung an Schwill schlagen Bleckmann und Pemberger (2021) für eine entwicklungsphasen-sensitive Medienerziehung drei Grundprinzipien vor: Erstens solle Medienerziehung in einem ersten Schritt mit analogen Medien und Techniken und erst später digital stattfinden, also «analog vor digital». Zweitens soll in den Lernszenarien das Produzieren dem Konsumieren vorausgehen. Drittens sollen erst Medien

5 Siehe auch: Initiative «CS unplugged - Computer Science without a computer» https://csunplugged.org/ de. 
gewählt werden, die eine möglichst vollständige Durchschaubarkeit der Funktionsweisen gewährleisten, bevor später «Black Boxes» ${ }^{6}$ wie Tablet/PC und Co. zum Zuge kommen (Bleckmann und Pemberger 2021).

\section{MünDig-Studie: Studiendesign, Messinstrumente, Stichprobenbeschreibung}

\subsection{Ziele und Studiendesign der MünDig-Studie}

Ziel der quantitativ-explorativen MünDig-Studie ist es, an reformpädagogischen Bildungseinrichtungen einen Einblick in die medienerzieherische Praxis und medienbezogene Einstellungen aller beteiligten Akteurinnen und Akteure zu erlangen. Im Rahmen der Studie wurden neben erstens Schul-zweitens auch KiTa-Eltern, drittens KiTa-Fachkräfte, viertens Lehrkräfte, sowie fünftens Schülerinnen und Schüler ab 16 Jahren befragt. Den fünf Gruppen wurde ein in weiten Teilen identischer Fragebogen vorgelegt. Im Folgenden werden jedoch aufgrund der oben hergeleiteten Fragestellung ausschliesslich die Ergebnisse der Elternbefragung an reformpädagogischen Schulen berichtet. Im Rahmen der Elternbefragung wurde angestrebt, möglichst viele Erziehungsberechtigte für eine Teilnahme an der Onlinestudie zu gewinnen. Viele Ressourcen wurden im Rahmen der Teilnehmergewinnung darauf verwendet, einen etwaigen Coverage Error (Bandilla 2015) einer Onlinebefragung zur Medienerziehung in der bereits beschriebenen Grundgesamtheit möglichst gering zu halten. Die Erhebung erfolgte in Kooperation mit den Waldorf- und Montessori-Verbänden (MDD, DMG, DMV). Mittels E-Mailverteilerlisten, die im Rahmen dieser Kooperation zugänglich gemacht wurden, konnten Einrichtungen ermittelt und davon insgesamt 618 Schulen kontaktiert werden. Jede Schule erhielt eine Einladungs-E-Mail mit einrichtungsspezifischem Teilnahmelink und der Bitte um Weiterleitung an die Befragtengruppen. Zusätzlich wurde ein Grossteil der Schulen telefonisch über die Studie informiert. Der Befragungszeitraum erstreckte sich von September bis Dezember 2019 und konnte vor jedweden Massnahmen, im Zuge der durch das Coronavirus SARS-CoV-2 verursachten Pandemie, abgeschlossen werden. Da die Teilnahme an der MünDig-Studie freiwillig war und jederzeit abgebrochen werden konnte, gibt es in den Befragtenzahlen grössere Unterschiede je nach Position der betreffenden Frage im Fragebogen (vgl. Abschnitt 4.3).

6 Siehe auch: Netzwerkinitiative «Unblack the box - für einen (selbst)bewussten Umgang mit digitalen Datentechnologien in Bildungseinrichtungen» https://unblackthebox.org. 


\subsection{Vertiefende Beschreibung ausgewählter Messinstrumente}

Im Folgenden sollen Teile des Messinstruments detaillierter vorgestellt werden, die neuartig sind und eigens für die MünDig Studie entwickelt wurden. Andere Daten wie die ebenfalls erfassten demographischen Daten, Angaben zur eigenen Person, zum Familienstand, den eigenen Kindern und deren Alter, die Ausstattungsquoten mit und Nutzungsdauer von verschiedenen Bildschirmmedien für die eigene Person und für das «jüngste Kind» wurden mit gängigen Befragungsinstrumenten erfasst.

Die Studienteilnehmenden wurden anlässlich der zu Beginn des Fragebogens erfolgenden Abfrage des Alters ihres jüngsten Kindes, welches eine reformpädagogische Schule besucht, auf eine sich wiederholende Unterscheidung in den Formulierungen hingewiesen: Mit der Formulierung «Kinder» oder «die Kinder» (vgl. z. B. Abb. 2) werden allgemeinere pädagogische Einstellungen erfragt. Die Formulierung «lhr Kind» bezieht sich dagegen jeweils konkret auf das genannte jüngste Kind.

\subsubsection{Abfrage der Selbsteinschätzung technischer Fähigkeiten}

Die Befragten wurden gebeten, ihre technischen Fähigkeiten im Umgang mit digitalen Endgeräten einzuschätzen. Die Beantwortung erfolgte mittels einer visuellen Analogskala, deren Endpole nicht beschriftet waren. ${ }^{7}$

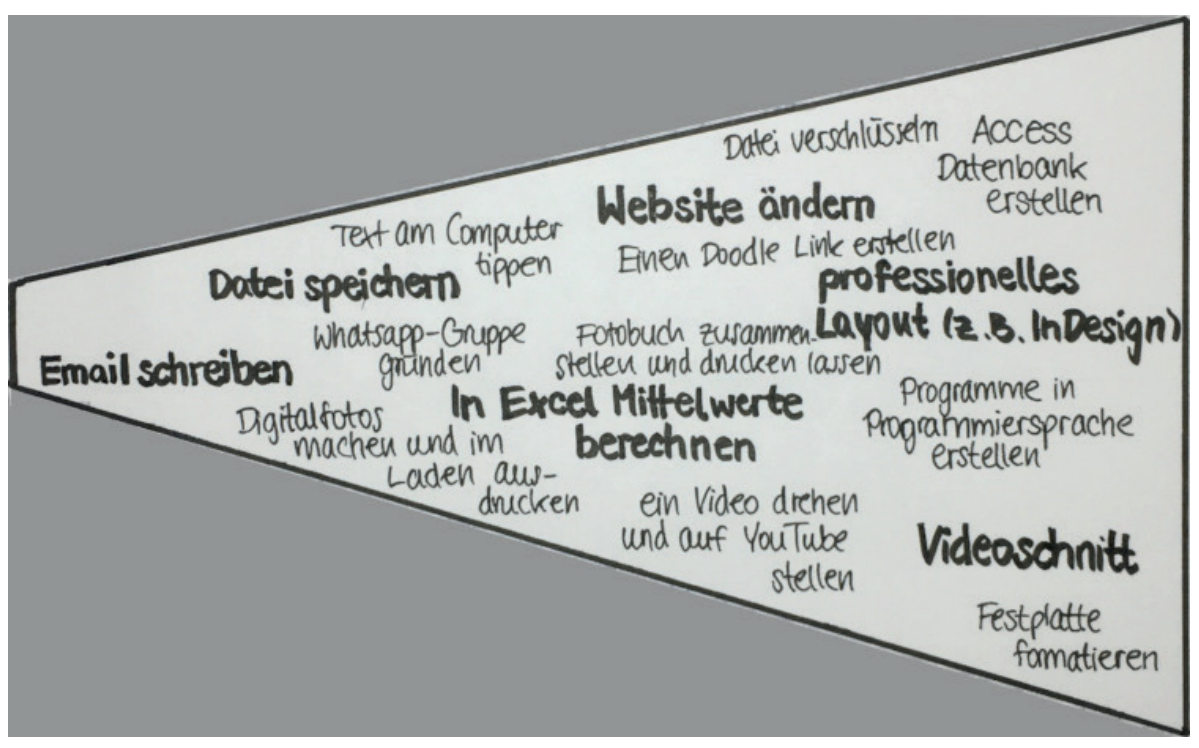

Abb. 1.: Bild zur Unterstützung der Auswahl des technischen Fertigkeitsniveaus.

7 «Wo würden Sie sich ungefähr einordnen bezüglich Ihrer Fähigkeiten zum Umgang mit digitalen Geräten? Die genannten Fähigkeiten sind nur Beispiele, es geht um Ihre Fähigkeit, eher simple, normale oder komplexe Anwendungen zu beherrschen.» 
Die Positionierung - weiter rechts oder links - der in Abb. 1 dargestellten Beispielaktivitäten erfolgte aufgrund eigener vorheriger Befragungen von tätigen und in Ausbildung befindlichen Pädagoginnen und Pädagogen (Bleckmann und J. Zimmer 2016), dabei hatten die Teilnehmenden die Möglichkeit, ihre Selbsteinschätzung von 1 (sehr niedrig, z. B. E-Mail schreiben) bis 6 (sehr hoch, z. B. Access Datenbank erstellen, Videoschnitt, Programme in Programmiersprache erstellen) abzugeben. Es kann angenommen werden, dass die medienbezogenen Einstellungen von pädagogischen Fachkräften und Eltern von deren technischen Fähigkeiten abhängen. Unklar ist dabei jedoch die ursächliche Richtung, bzw. kann zunächst ein beidseitiger Effekt angenommen werden: je mehr digitale Fähigkeiten, desto stärker tendieren Befragte zu einer frühen und ausgeprägten Befürwortung digitaler Aktivitäten, oder sie könnten diese auch und gerade in Kenntnis der Risiken (vgl. oben: Silicon Valley Eltern) gerade vermehrt erst in späterem Alter oder insgesamt weniger für sinnvoll halten.

\subsubsection{Doppelschieberegler - Welches Medium in welchem Alter für welchen Zweck?}

Für eine Abfrage medienbildnerischer Einstellungen ${ }^{8}$ in drei im Folgenden genannten Subdimensionen wurde ein neues Befragungsinstrument erstellt. Auf Objektebene wird dabei eine latente Dimension in Form von entwickelten Überzeugungen/Dispositionen von pädagogischen Fachkräften und Eltern angenommen, die sich in dem unterschiedlichen Befürworten bzw. Ablehnen von medienbildnerischen Aktivitäten, mit bzw. ohne Einsatz digitaler Bildschirmmedien ausdrücken. Weiter wird davon ausgegangen, dass diese dazu führen, dass bestimmte medienbildnerische Aktivitäten in pädagogischen Settings eher befürwortet und für sinnvoll erachtet werden. Das Befürworten von medienerzieherischen Aktivitäten mit/ohne Bildschirm wird dabei als eine erste Subdimension konzeptualisiert. Der zu identifizierende Zweck/ das Lernziel der Aktivität -abgefragt in den 10 Bereichen gemäss Tabelle 1 - sowie die Altersspanne der Schülerinnen und Schüler, mit denen derartige Aktivitäten durchgeführt werden, stellen zwei weitere Subdimensionen der angenommenen Disposition dar, deren Ausprägung unabhängig voneinander sein kann.

8 In der Elternbefragung wurde der Doppelschieberegler nur für die Abfrage der als sinnvoll erachteten Aktivitäten verwendet (Einstellungsebene), in der Lehrkräftebefragung wurde derselbe Schieberegler nochmals zur Abfrage der medienbildnerischen Praxis verwendet, womit ein direkter Abgleich zwischen «Ist» (Praxis) und «Soll» (Einstellung) ermöglicht werden soll. Dabei wird angenommen, dass medienbildnerische Einstellungen einen entscheidenden Einfluss darauf ausüben, welche medienbildnerischen Aktivitäten pädagogische Fachkräfte für welchen Zweck und für welche Altersspanne der Educandi für sinnvoll erachten. Des Weiteren kann begründet angenommen werden, dass medienerzieherische Einstellungen einen Einfluss auf die tatsächliche medienerzieherische Praxis der Educanten ausüben (hierzu berichten wir jedoch im vorliegenden Beitrag keine Ergebnisse). 


\begin{tabular}{|l|l|}
\hline \multicolumn{1}{|c|}{6 Bereiche des Medienkompetenzrahmens } & \multicolumn{1}{c|}{ Zusätzliche 4 Bereiche } \\
\hline - Bedienen und Anwenden & - Medieneinsatz durch Fachkräfte \\
- informieren und Recherchieren & - Elternzusammenarbeit \\
- Kommunizieren und Kooperieren & - Ressourcenorientierte \\
- Produzieren und Präsentieren & - Mediensuchtprävention \\
- Analysieren und Reflektieren & - Unterstützung bei der Verarbeitung von \\
- Problemlösen und Modellieren & Medienerlebnissen \\
\hline
\end{tabular}

Tab. 1.: 10 Bereiche der Medienbildung im Kontext von Schule.

Die ersten sechs Bereiche entsprechen den sechs übergeordneten Kompetenzbereichen von Schülerinnen und Schülern im Medienkompetenzrahmen NRW, ergänzt um weitere 4 Bereiche, von denen die letzten drei aufgrund qualitativer Vorstudien an reformpädagogischen Bildungseinrichtungen, sowie Expertinnen- und Experteninterviews hinzugenommen wurden.

Um eine solche multidimensionale Abfrage, in der für jeden der 10 Bereiche noch 6 Beispielaktivitäten ${ }^{9}$ genannt wurden, für die Befragten mit vertretbarem Zeitaufwand bewältigbar zu gestalten, wurde in Zusammenarbeit mit Soscisurvey, dem ausgewählten Anbieter für die Onlinebefragung, ein Doppel-Schieberegler eigens programmiert (vgl. Abb. 2). Es handelt sich hier anders als in vorigen Versuchen einer altersdifferenzierten Erfassung (Bitzer, Bleckmann, und Mößle 2014) nicht um eine «künstliche» Antwortskalierung in vorgegebene Altersrubriken (z. B. «Krippenalter» «Kindergartenalter» «Grundschulalter», ...), sondern um die alltagsweltlich relevante Altersspanne 0-18 Jahre. Anfangs erscheint den Befragten eine visuelle Analogskala in hellgrau. Mit der Einstellung eines Reglers (Markierung zu Einstiegs- oder Ausstiegsalter) wird automatisch die Spanne zum jeweils anderen Regler dunkler und breiter eingefärbt. Beide Reglermarken werden automatisch mit dem eingestellten Alter beschriftet. Die eingestellte Altersspanne kann beliebig oft verändert werden.

9 In den Bereichen 1 bis 7 und 10 wurde zusätzlich zwischen Aktivitäten mit und ohne Einsatz digitaler Bildschirmmedien unterschieden. Eine derartige Definition in Beobachtungssprache zu übersetzen ist zumindest fehleranfällig. Aus diesem Grund wurde im Rahmen der Studie jeweils zuerst ein beispielorientiertes Vorgehen gewählt, um die Beantwortung auch für Nicht-Fachkräfte (Eltern, Schülerinnen und Schüler) zu verbessern. Andererseits wurde mit der reinen Anzahl an Items sichergestellt, dass die Indikatoren-Stichprobe möglichst gross ausfällt, um eine Unkenntnis der abgefragten Beispiele ausgleichen zu können. 


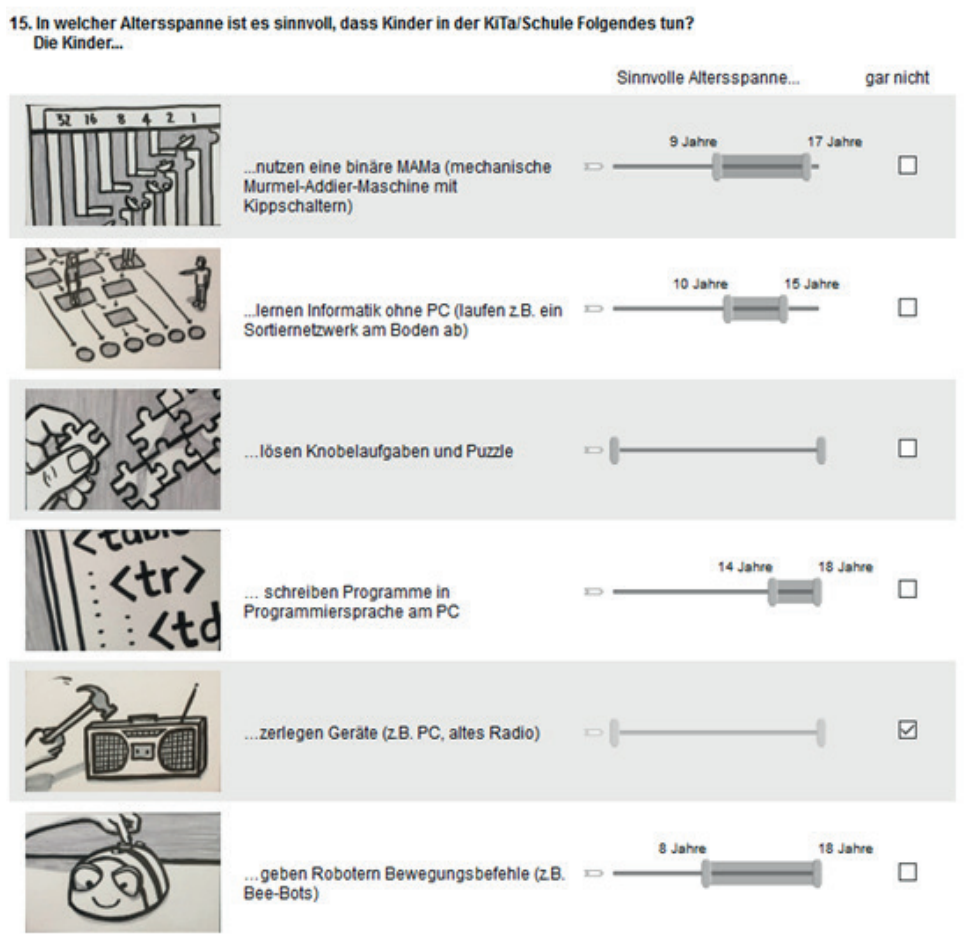

Abb. 2.: Screenshot Doppelschieberegler-Abfrage Bereich Problemlösen und Modellieren.

Da die Fragebogentestenden vermehrt trotz der erheblichen Zeitersparnis durch den Doppelschieberegler im Vergleich zum Ankreuzen von Alterskategorien immer noch über einen zu langen und repetitiv wirkenden Fragebogen klagten, wurden die insgesamt 60 abgefragten Beispielaktivitäten mit Grafiken illustriert. Damit konnten z.T. die Item-Texte kürzer gefasst werden und die Motivation der Befragungsteilnehmer nochmals deutlich erhöht werden. Die Verwendung von Grafiken in quantitativen Befragungsinstrumenten wird bisher trotz einiger Vorteile (Couper 2008; Bandilla 2015) noch selten umgesetzt, wobei eine einheitliche Gestaltung der Grafiken wichtig ist, um das Antwortverhalten nicht zu beeinflussen. Daher wurden alle Grafiken im selben schwarz-weiss gezeichneten Stil durch eine Projektmitarbeiterin eigens angefertigt.

\subsubsection{Elternzufriedenheit mit der medienbildnerischen Praxis}

Die Elternzufriedenheit mit der medienbildnerischen Praxis an ihrer reformpädagogisch orientierten Schule wurde auf zweierlei Weise erfragt: Einmal für jeden der 10 Bereiche direkt nach der Abfrage dessen, was als sinnvoll erachtet wurde, sowie am Ende der Befragung nochmals bereichsübergreifend. 
Die bereichsspezifische Abfrage der Elternzufriedenheit erfolgte mit folgender Formulierung: «Bitte geben Sie an, ob Ihr Kind Ihrer Meinung nach auch über die abgefragten Beispielaktivitäten hinaus im Unterricht folgendes zu selten, genau richtig, häufig oder zu oft tut». Als vierte (Ausweich-)Option bestand die Möglichkeit, «weiß nicht» anzukreuzen. Die Item-Formulierung der Antworten für Bereiche 1 bis 7 und 10, hier für Bereich 1, lauteten: «Produzieren und Präsentieren mit Medien ohne Bildschirm» vs. «Produzieren und Präsentieren mit Medien mit Bildschirm». Im Bereich 8 wurde nach der Zufriedenheit mit der Häufigkeit von Elternzusammenarbeit («pädagogische Unterstützung» vs. «technische Unterstützung») gefragt, in Bereich 9 nach der Stärkung von Kindern im Leben zum Schutz vor Digital-Risiken «als Einzelpersönlichkeit» vs. «im sozialen Miteinander». Da die Angaben, ob die jeweiligen Aktivitäten nach Einschätzung der Eltern zu selten, genau richtig, häufig oder zu oft umgesetzt werden, noch keinen direkten Rückschluss auf die Zufriedenheit zulässt (es könnte auch etwas zwar mit der richtigen Häufigkeit, aber einer aus Sicht der Eltern mangelhaften Umsetzung und damit verbundener hoher Unzufriedenheit erfolgen), wurde zur Konstrukt-Validierung eine zusätzliche Frage mit einer direkten Abfrage der Zufriedenheit hinzugenommen, mit folgender Formulierung:

"Jetzt noch einmal allgemeiner gefragt: Wie zufrieden sind sie mit der Medienerziehung an der Schule Ihres Kindes, was die folgenden übergreifenden Aspekte angeht: Langfristig Medienmündigkeit der Schüler*innen fördern durch...». (Antwortoptionen siehe Ergebnisteil Abb. 3).

\subsection{Stichprobenbeschreibung}

Die erste inhaltliche Frage beantworteten 2.933 Erziehungsberechtigte ${ }^{10}$, die letzte Frage 1.252 Erziehungsberechtigte. Daraus resultiert eine Beendigungsquote von 42,7 Prozent. Die durchschnittliche Dauer für die Bearbeitung des gesamten Fragebogens lag bei den Schuleltern bei knapp unter einer halben Stunde, mit Schwankungen zwischen knapp einer Viertelstunde und über einer Dreiviertel-Stunde.

10 Durch einen erst im Oktober 2021 behobenen Fehler in der Zusammenführung der beiden Datensätze der KiTa- und der Schulbefragung sind in der diesem Artikel zugrunde liegenden ersten Auswertung der Daten nur die Antworten von $n=2.933$ Eltern einbezogen worden. Inzwischen wurde der Fehler behoben und es liegen Daten zu n = 3.149 Schuleltern vor (und zu n = 2.172 KiTa-Eltern, über die erste Ergebnisse an anderer Stelle publiziert wurden (Bleckmann, Denzl, und Streit 2021; Kernbach und Streit 2021). 


\begin{tabular}{|l|l|c|c|}
\hline \multicolumn{2}{|c|}{} & Absolut & In $\%$ \\
\hline \multirow{4}{*}{ Schulform } & Montessori(orientiert) & 524 & 17,9 \\
\cline { 2 - 4 } & Waldorf & 2381 & 81,2 \\
\cline { 2 - 4 } & Anderer reformpädagogischer Ansatz & 28 & 1 \\
\hline \multirow{5}{*}{ Geschlecht } & weiblich & 1214 & 72,0 \\
\cline { 2 - 4 } & männlich & 453 & 26,9 \\
\cline { 2 - 4 } & anderes & 6 & 0,4 \\
\cline { 2 - 4 } & keine Angabe & 12 & 0,7 \\
\hline \multirow{5}{*}{ Alter } & $1-30$ Jahre & 21 & 1,2 \\
\cline { 2 - 4 } & $31-40$ Jahre & 470 & 27,6 \\
\cline { 2 - 4 } & $41-50$ Jahre & 859 & 50,4 \\
\cline { 2 - 4 } & $51-60$ Jahre & 332 & 19,5 \\
\cline { 2 - 4 } & $61-70$ & 13 & 0,8 \\
\cline { 2 - 4 } & Über 70 & 2 & 0,1 \\
\hline \multirow{5}{*}{ Erwerbstätigkeit } & nicht erwerbstätig & 152 & 10,8 \\
\cline { 2 - 4 } & erwerbstätig in Teilzeit & 536 & 50,9 \\
\cline { 2 - 4 } & Erwerbstätig in Vollzeit & & 38,2 \\
\hline
\end{tabular}

Tab. 2.: Soziodemographische Merkmale der selbstrekrutierten Stichprobe.

Für die Frage nach dem «jüngsten Kind» (s.o.) ergeben sich wie erwartet deutliche Unterschiede innerhalb der Stichprobe: Fast die Hälfte der Befragten gibt an, dass dieses Kind die Klasse 1-3 besucht (44,8\%). Beinahe jede/r vierte Befragte ist Erziehungsberechtigte/r eines Kindes, das die Klassen 4-6 besucht (24,8 \%). Die Klassen 7-9 besuchen die jüngsten Kinder von 18,6 Prozent der Befragten, während von rund 12 Prozent der Befragten das jüngste Kind die Klassen 10-13 besucht (11,9 \%).

Die Selbsteinschätzung der Eltern an reformpädagogischen Schulen bezüglich ihrer technischen Fähigkeiten im Umgang mit digitalen Endgeräten ist mit einem Mittelwert von 3,9 insgesamt als «mittel» zu bezeichnen.

\section{Ergebnisse}

\subsection{Elternzufriedenheit im Überblick}

Im Rahmen der MünDig-Studie wurden Erziehungsberechtigte befragt, wie zufrieden sie mit verschiedenen Aspekten der schulischen medienerzieherischen Praxis sind. Die Zufriedenheit wurde dabei zweiteilig erfasst. Bevor im Fragebogen eine allgemeine Zufriedenheit mit der medienerzieherischen Praxis erfasst wurde, konnten Erziehungsberechtigte differenzierte Bewertungen zu zehn verschiedenen Bereichen der Medienerziehung abgeben. 
Einleitend wird der Blick auf die globale Zufriedenheit der Erziehungsberechtigten geworfen. Diese setzt sich aus der Bewertung von vier Aktivitätsbereichen zusammen, in denen Schulen Medienmündigkeit fördern: Förderung durch Einsatz von Medien mit und ohne Bildschirm, durch die medienerzieherische/medienpädagogische Zusammenarbeit mit dem Elternhaus und durch eine Pädagogik, die zu kritischem und eigenständigem Denken und Handeln anregt. Die Angabe der Beurteilung folgte jeweils auf einer 4-stufigen Antwortskala von (1) «überhaupt nicht zufrieden» bis (4) «sehr zufrieden» (siehe Kapitel 4).

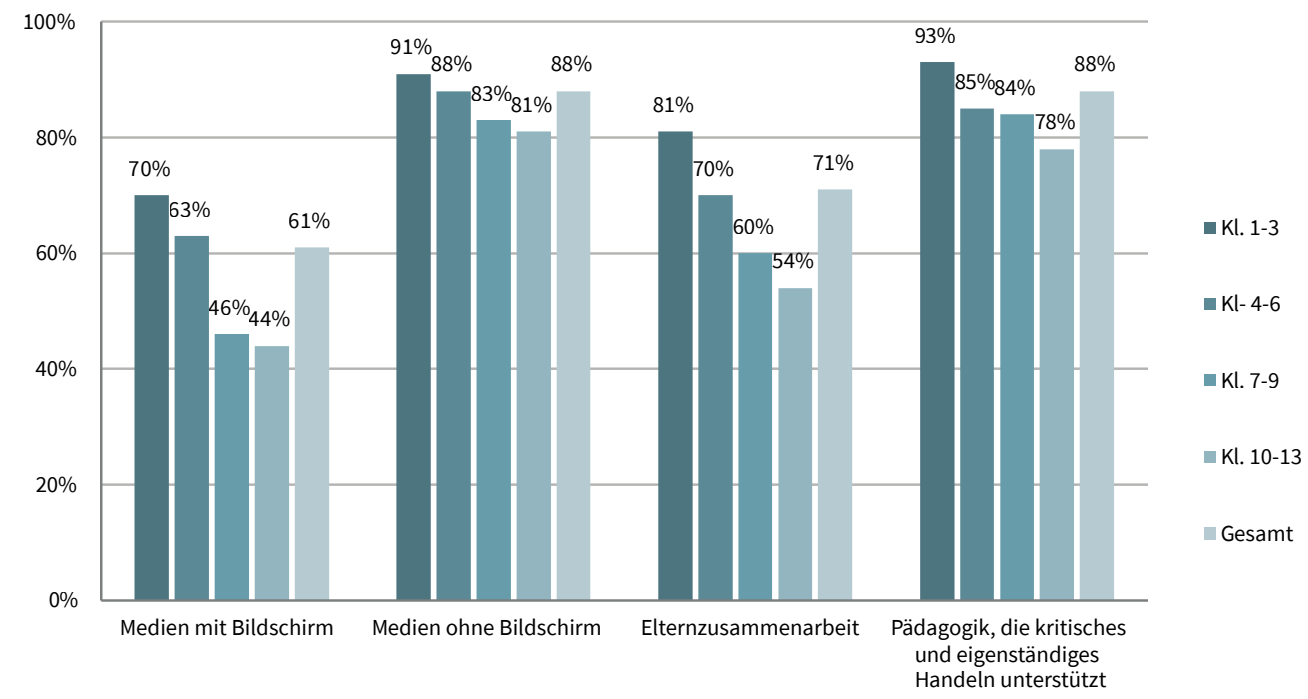

Abb. 3.: Zufriedenheit mit allgemeiner Medienerziehung (in \%, kumulierter Anteil eher und sehr zufrieden). Fallzahlen: Kl. 1-3, N = 718-755; Kl. 4-6, N = 392-403; Kl. 7-9, N = 326-330; Kl. 10-13, N = 186-191 | Medien mit Bildschirm: Gesamt, N = 1622; Medien ohne Bildschirm: Gesamt, N = 1675; Elternzusammenarbeit: Gesamt, N = 1671; Pädagogik, die...: Gesamt, $\mathrm{N}=1674$.

Insgesamt zeigen die Ergebnisse der MünDig-Studie, dass Erziehungsberechtigte der medienerzieherischen Praxis an reformpädagogischen Schulen ein positives Zeugnis ausstellen (s. Abb. 1). Rund 88 Prozent der Befragten sind mit der Förderung von Medienmündigkeit durch den unterrichtlichen Einsatz von Medien ohne Bildschirm und einer Pädagogik, die kritisches und eigenständiges Denken und Handeln unterstützt, zufrieden. 71 Prozent der Befragten sind mit der Elternzusammenarbeit zufrieden. ${ }^{11}$ Der Einsatz von Medien mit Bildschirm wird von mehr als der Hälfte als positiv bewertet (61\%). Um sich einer Bewertung der altersspezifischen Praxis zu nähern, wurden die Daten anhand von vier Alterskategorien aufgeteilt (s. Abb. 1). In allen vier abgefragten Bereichen zeigt sich dabei der gleiche Trend: Erziehungsberechtigte, deren Kinder jünger sind, sind häufiger zufrieden als Erziehungsberechtigte älterer Kinder. In jedem Fall ist der Anteil zufriedener Eltern in den Klassenstufen

11 Kumulierter Anteil der Antworten (3) «eher zufrieden» und (4) «sehr zufrieden». 
1-3 grösser als im Gesamtdurchschnitt. Unter den Erziehungsberechtigten, die ihre Angaben auf Jugendliche der Klassen 10-13 beziehen, fällt die Zufriedenheit geringer aus. Dies gilt insbesondere für Aktivitäten mit Bildschirmmedien (44 \%) und für die medienerzieherische/medienpädagogische Elternzusammenarbeit (54\%). Die geringsten altersspezifischen Unterschiede finden sich bei der Bewertung einer Pädagogik, die kritisches und eigenständiges Denken und Handeln fördert sowie der Medienerziehung mit Medien ohne Bildschirm. In diesen beiden Bereichen ist über alle Altersstufen hinweg ein grosser Anteil der Befragten $(>=78 \%)$ zufrieden mit der medienerzieherischen Praxis an der reformpädagogischen Schule ihrer Kinder.

Über die oben berichtete, globale elterliche Zufriedenheit hinaus wurden Erziehungsberechtigte in der MünDig-Studie um weitergehende Bewertungen der medienerzieherischen Praxis gebeten. Es werden dabei insgesamt zehn verschiedene medienerzieherische Zieldimensionen unterschieden. Die ersten sechs sind an den sechs Kompetenzbereichen des Medienkompetenzrahmens NRW (s. Abschnitt 1.1) orientiert. Darüber hinaus wird eine Bewertung des Medieneinsatzes der Lehrkräfte im Unterricht abgefragt. Abschnitt 5.1.2 ist anschliessend den eigens für die MünDig-Studie entwickelten weiteren Bereichen vorbehalten: Elternzusammenarbeit, ressourcenorientierte Mediensuchtprävention und Verarbeitung belastender Medienerlebnisse (s. Abschnitt 3.1). Die Befragten werden in jedem Kompetenzbereich lernzielbezogen befragt, wie sie die Häufigkeit der unterrichtlichen Bearbeitung bewerten. Dabei stehen die Antwortkategorien (1) «zu selten» (2) «genau richtig» oder (3) «zu oft» zur Verfügung. Eine Ausweichkategorie ist mit «weiss nicht» gegeben. Die ersten sieben Kompetenzbereiche medienerzieherischer Praxis werden nach Medien mit und ohne Bildschirm unterschieden (vgl. Abb. 2). In Übereinstimmung mit der vorherigen globalen Abfrage, zeigt sich auch in dieser differenzierten Abfrage insgesamt ein hoher Anteil an zufriedenen Erziehungsberechtigten.

Das Verfolgen aller Kompetenzbereiche mit Medien ohne Bildschirm bewertet jeweils mehr als die Hälfte der Befragten als «genau richtig». Insbesondere die Häufigkeit der Tätigkeiten der Schülerinnen und Schüler in den Bereichen Produzieren und Präsentieren mit Medien ohne Bildschirm (68,7\%), Bedienen und Anwenden von Medien ohne Bildschirm $(67,5)$, sowie der Einsatz bildschirmfreier Medien durch die Lehrkräfte $(71,7 \%)$ wird durch die Eltern grösstenteils befürwortet. Allerdings sei abschliessend darauf hingewiesen, dass die ermittelten Ergebnisse bislang noch keine Aussagen darüber zulassen, wie häufig die abgefragten Aktivitäten in der pädagogischen Alltagspraxis tatsächlich bearbeitet werden. Die Bewertung "genau richtig» kann durchaus bedeuten, dass der Einsatz von Medien mit/ohne Bildschirm de facto sehr häufig oder sehr selten stattfindet. Weitere Analysen der vorliegenden Daten können und sollten diese offene Frage zukünftig klären, hierfür bieten sich auch altersspezifische Analysen an, die in Abschnitt 6.2 exemplarisch ausgeführt werden. 


\subsubsection{Produzieren und Präsentieren mit Medien mit und ohne Bildschirm}

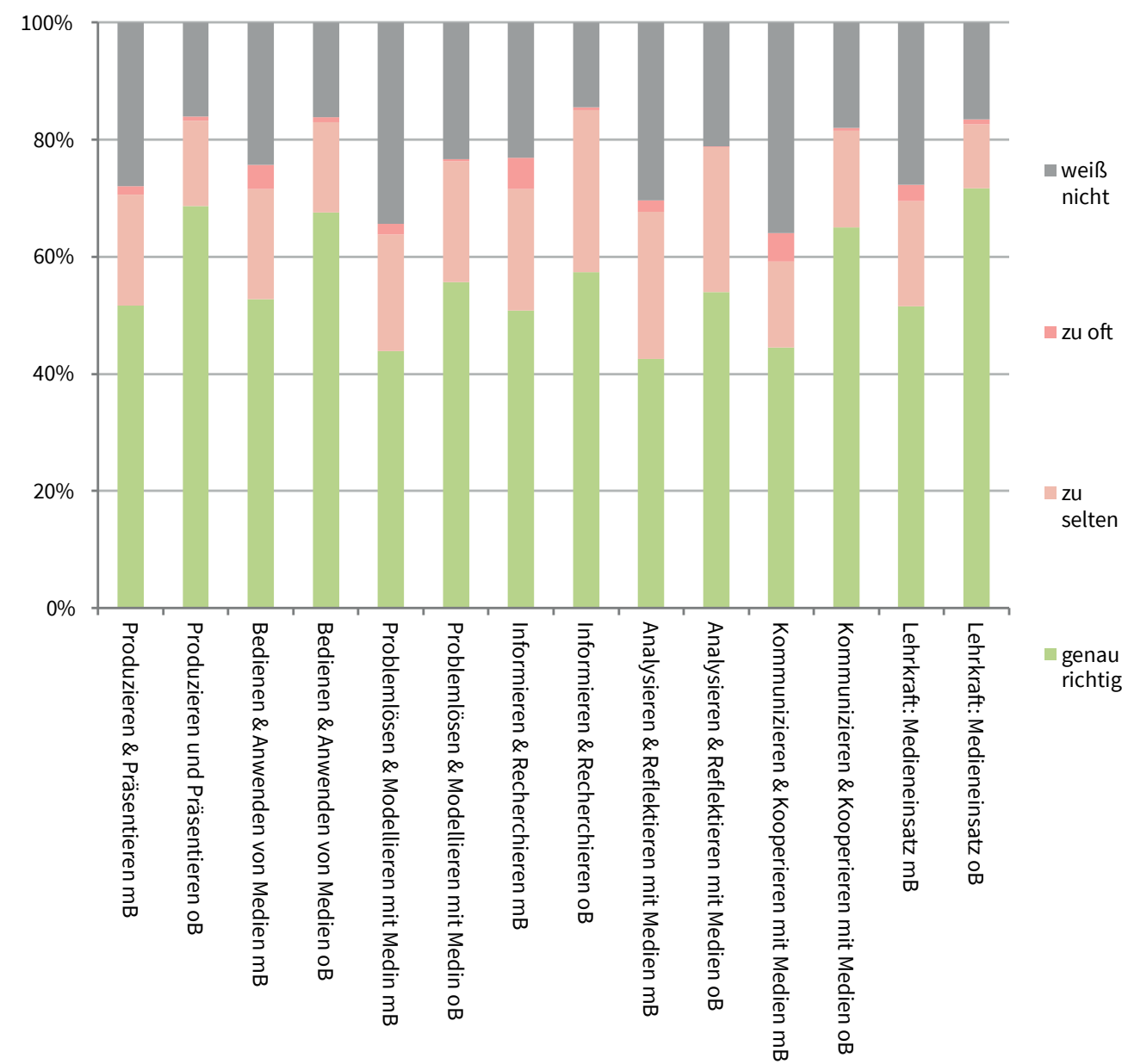

Abb. 4.: Elternzufriedenheit hinsichtlich der ersten sieben abgefragten Bildungsziele (in \%), $\mathrm{mB}=$ mit Bildschirm; $\mathrm{oB}=$ ohne Bildschirm; Fallzahlen siehe Tabelle 1, Anhang.

Für die zehn verschiedenen Kompetenzbereiche wurden Eltern zuerst gefragt, für welche Altersspanne sie eine ausgewählte Beispiel-Aktivität für sinnvoll erachten (vgl. Abschnitt 4.2). Mit zwei Schiebereglern können Beginn (im Folgenden Einstiegsalter) und Ende (im Folgenden Ausstiegsalter) dieser Spanne auf einer gegebenen Skala von 0 bis 18 Jahren angegeben werden. Für jeden Bereich wurden 6 verschiedene Beispiel-Aktivitäten genannt, wovon in den meisten Bereichen je drei mit und drei ohne Einsatz von Bildschirmmedien ausgewählt waren. Im Folgenden werden die Ergebnisse nur für einen der zehn Bereiche, namentlich die Schülerinnen- und Schüleraktivität «Produzieren und Präsentieren» ausführlich dargestellt. Detaillierte und vollständige Ergebnisse sind in den Studienberichten einzusehen (Bleckmann et al. 2021b im Erscheinen, 2021a im Erscheinen). 


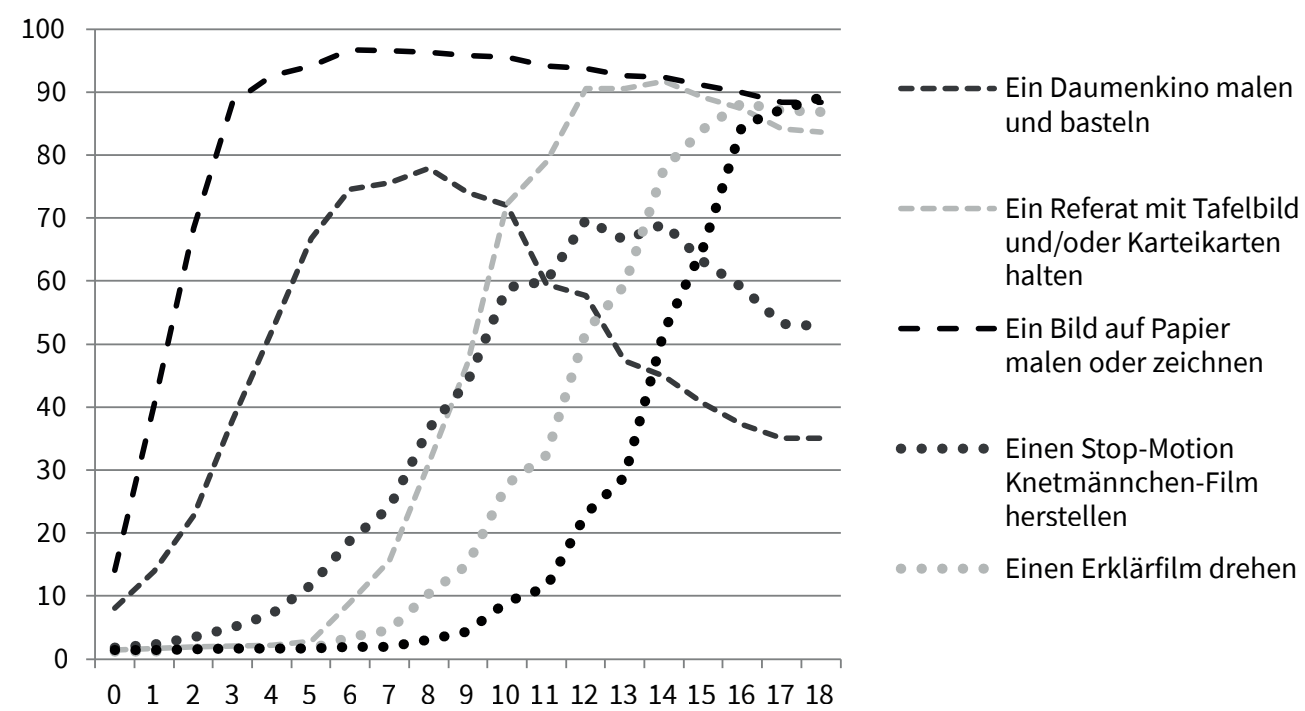

Abb. 5.: Sinnvolle Medienaktivität nach Alter (in \%); Fallzahlen: Daumenkino malen und basteln: $\mathrm{N}=2402$; Referat mit Tafelbild und/oder Karteiarten halten: $\mathrm{N}=2315$; Bild auf Papier malen oder zeichnen: $\mathrm{N}=2190$; Ein Stop-Motion-Knetmännchen-Filmherstellen: $\mathrm{N}=2324$; Erklärfilm drehen: $\mathrm{N}=2307$; Webiste erstellen: $\mathrm{N}=2187$.

Um die im ausgewählten Bereich ermittelten Ergebnisse zu berichten, werden diese für einen Vergleich der Aktivitäten im Bereich «Produzieren und Präsentieren mit und ohne Bildschirm» folgendermassen aufbereitet: Zunächst dient das durchschnittliche Einstiegsalter, ab dem die abgefragte Aktivität als sinnvoll erachtet wird, als erster Referenzwert. Aus einem derartigen Verlauf relativer Häufigkeit ergibt sich ein Überblick, welche Aktivitäten früher/später, altersklassenspezifisch oder -übergreifend als sinnvoll erachtet werden. Die befragten Erziehungsberechtigten geben für die Aktivitäten ohne Bildschirm im Bereich «Produzieren und Präsentieren» ein deutlich niedrigeres Einstiegsalter an, ab dem die jeweilige Aktivität als sinnvoll erachtet wird, als für Aktivitäten mit dem Einsatz von Bildschirmmedien. Das durchschnittliche Einstiegsalter für «Daumenkino» liegt bei 4,8 Jahren $(S D=3,6)$, für «Referat mit Tafelbild und/oder Karteikarten halten» bei 9,4 $(S D=2,5)$ und für «Ein Bild auf Papier malen oder zeichnen» bei 2,2 (SD = 2,3). Für die Aktivitäten mit Bildschirm liegen die durchschnittlichen Einstiegsalter höher: «Einen Stop-Motion-Knetmännchen-Film herstellen» halten Befragte im Durchschnitt ab 10 Jahren $(S D=4,4)$ für sinnvoll. «Einen Erklärfilm drehen» wird ab durchschnittlich 12,4 Jahren $(S D=3,3$ ) und «Eine Website erstellen» ab 14,2 Jahren $(S D=3)$ als medienpädagogisch sinnvolle Aktivität erachtet. Darüber hinaus wird die jeweilige Altersspanne fokussiert, die die Befragten für ein sinnvolles Alter bewerten. Konkret wird ermittelt, wie viele Befragte ein jeweiliges Alter (0-18 Jahre) innerhalb ihrer als sinnvoll angegebenen Altersspanne angeben. Abb. 3 zeigt dazu für jedes Alter (0-18 Jahre) an, wie viele Befragte dieses innerhalb einer sinnvollen Altersspanne einordnen. Die Übersicht dient 
insbesondere dem Vergleich der Ergebnisse zwischen den Aktivitäten mit und ohne Bildschirm. ${ }^{12}$ Dabei ist festzuhalten, dass die Beispiel-Aktivitäten ohne Bildschirm (gestrichelt) häufiger für jüngere Altersgruppen als sinnvoll erachtet werden als diejenigen mit Bildschirm (gepunktet). Zudem zeigt sich, dass «ein Bild auf Papier zeichnen oder Malen» und "einen Stop-Motion-Knetmännchen-Film» herstellen die zwei einzigen Aktivitäten sind, die mit zunehmendem Alter der Kinder weniger häufig als sinnvoll erachtet werden. Die anderen vier Aktivitäten erachten viele Befragte über das 18. Lebensjahr hinaus als sinnvoll.

\subsubsection{Elternzusammenarbeit, ressourcenorientierte Prävention und Verarbeitungshilfen bei Digital-Risiken}

Im Bereich der Unterstützung für Eltern in der Medienerziehung durch die Schule, wie auch im Bereich der ressourcenorientierten Prävention von Digital-Risiken, werden die Beispiel-Items über ein sehr breites Altersspektrum als sinnvoll angesehen (Bleckmann et al. 2021b im Erscheinen, 2021a im Erscheinen).

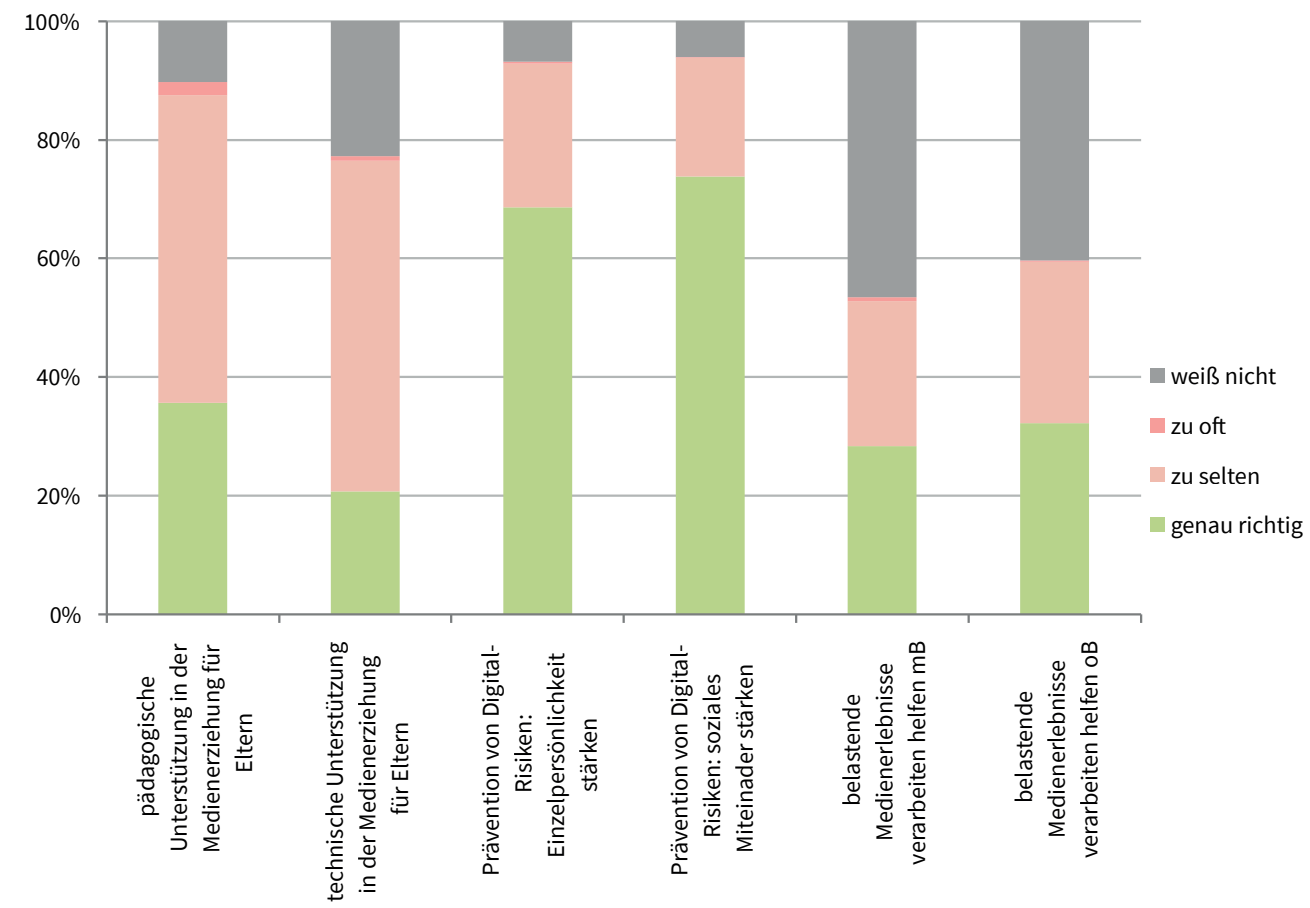

Abb. 6.: Elternbewertung der medienerzieherischen Tätigkeiten Bereiche 8-10; «mB» = mit Bildschirm; «OB» = ohne Bildschirm; Fallzahlen siehe Tabelle 1, Anhang.

12 Aufgrund einer besseren Lesbarkeit wird auf die Angabe der einzelnen Werte verzichtet. Die Abbildung dient im Rahmen der explorativen Studie einer ersten Annäherung und Evaluation der Messinstrumente. Die einzelnen Werte sind Tab. 2 im Anhang zu entnehmen. 
Mit der Häufigkeit der tatsächlichen Umsetzung sind die Eltern im Bereich der Prävention über alle Altersstufen hinweg überwiegend zufrieden. Die Einschätzung, dies werde "genau richtig» umgesetzt, liegt je nach Klassenstufe zwischen $63 \%$ und $77 \%$, mit etwas weniger positiven Bewertungen für die Stärkung der Eigenpersönlichkeit (im Mittel: $68 \%$ ) als für die Stärkung des sozialen Miteinander (im Mittel $74 \%)$ und geringfügig niedrigeren Werten für die älteren Klassenstufen. Dagegen ist der Bereich der Elternzusammenarbeit mit Abstand derjenige, in dem die Eltern am häufigsten eine «zu seltene» Umsetzung bemängeln. Dies trifft bereits für die pädagogische Unterstützung zu (43 \% - $64 \%$ «zu selten», Beispiel-Items waren hier u. a. «Eltern anregen, im Beisein des Kindes auf die eigene Mediennutzung zu achten» und «Eltern unterstützen, Freizeit mit ihrem Kind ohne Bildschirm zu gestalten»), und noch stärker und mit dem Alter stärker zunehmend auf die technische Unterstützung (45 \% - $71 \%$ «zu selten», Beispiel-Item «Eltern bei der Installation von Zeitbegrenzungs- und Filtersoftware unterstützen»). Was die Unterstützung bei der Verarbeitung belastender Medienerlebnisse angeht, so haben hier auffällig viele Eltern «weiss nicht» angegeben, in den unteren Klassen sogar mehr als die Hälfte. Die übrigen Eltern sind zu etwa der Hälfte der Meinung, dies geschehe "genau richtig», wobei auch dies mit steigender Klassenstufe seltener angegeben wird.

\section{Diskussion und Ausblick}

\subsection{Limitationen der Aussagekraft der Studienergebnisse}

Die Beendigungsquote ist mit über $40 \%$ für eine Befragung mit einer Gesamtdauer von ca. 30 Minuten (für Eltern, bei den pädagogischen Fachkräften mit ca. 45 Minuten noch höher) bemerkenswert hoch, was für eine gute Passung zwischen Befragungsinstrument und Zielgruppe spricht. Dennoch ist die Rücklaufquote für eine Vollerhebung mit knapp 3000 von geschätzten über 100000 Elternhäusern sicher unter $3 \%$ und damit insgesamt so niedrig, dass die Ergebnisse zunächst nur bedingt auf die Gesamtheit aller Eltern an reformpädagogischen Schulen übertragen werden können. Darüber hinaus ist ein Abgleich der Stichprobe mit der theoretisch definierten Grundgesamtheit kaum möglich, da reformpädagogische Schulen nicht immer klar von staatlichen Regelschulen zu trennen sind. Es kann durch die Onlinebefragung kein repräsentatives Sampling-Verfahren angewendet werden. Eine Möglichkeit, in Zukunft zumindest etwas mehr über die Repräsentativität aussagen zu können, bietet der Vergleich zwischen den Gesamtergebnissen der Studie und den Ergebnissen aus einzelnen Schulen, in denen die Rücklaufquoten sehr viel höher lagen, mit Beteiligung von zum Teil etwa der Hälfte der Elternhäuser. Dieser Abgleich ist bisher noch nicht erfolgt. 
Weniger technik-affine Elternhäuser dürften aufgrund des Online-Formates unterrepräsentiert sein, während eine Überrepräsentation der sich durch den Studientitel angesprochen gefühlten Eltern nahe liegt. «MünDig» im Titel der Studie als Akronym für Mündigkeit und Digitalisierung bzw. der in der Studie verwendete Begriff «Medienmündigkeit» nach dem Werk von Bleckmann (2020) ist in reformpädagogischen Kreisen geläufig. Dagegen spricht allerdings, dass sich der Begriff heute u. a. aufgrund der Verwendung durch die Kinderkommission im Deutschen Bundestag (Wiesmann 2019) und Bernhard Pörksen (2018) im Sprachgebrauch etabliert hat.

Da die Differenzierung in «Medien mit Bildschirm» und «Medien ohne Bildschirm» in Kombination mit vorher abgefragten konkreten Lernszenarien, bei deren Abfrage bewusst zwischen Szenarien mit und ohne Bildschirm abgewechselt wurde, ohne diese Unterscheidung explizit zu benennen (vgl. Abschnitt 5.1.1) von den Befragten gut verstanden und angenommen wurde, kann diese Differenzierung nicht als Studienlimitation betrachtet werden. Die Gründe dafür, weshalb Befragte mitunter in grossen Teilen mit der Ausweichkategorie «weiss nicht» geantwortet haben, bleiben zu eruieren. Fehlt Erziehungsberechtigten die Kenntnis der medienerzieherischen Praxis oder haben sie medienbezogene Einstellungen nicht in der abgefragten Differenzierung ausgebildet? In weiteren Analysen und Folgestudien sollte dieser Frage nachgegangen werden. Ein zweistufiges Verfahren, in dem zunächst abgefragt wird, ob bestimmte Zusammenarbeiten stattfinden, die im zweiten Schritt bewerten werden können, würde Erkenntnisse dazu bereitstellen. Es ist zu betonen, dass die entwickelte Abfrage medienbezogener Einstellungen im Rahmen der «sinnvollen Altersspanne» Beispiel-Aktivitäten erfragt. Eine Evaluation unter Rückgriff auf verallgemeinerbare Abfragen auf Einstellungsebene bleibt bislang noch aus.

Da die MünDig-Studie eine explorative Studie ist, in der viele neu entwickelte Befragungsinstrumente zum Einsatz kommen, ist eine Vergleichbarkeit mit anderen Studien nicht zu erbringen. Die hier berichteten Ergebnisse beschreiben auf Grundlage deskriptiver Daten Einstellungen und Handlungsweisen der Befragten, liefern jedoch keine Erklärungsmodelle für Zusammenhänge zwischen diesen Daten.

Die Charakterisierung verschiedener Untergruppen mit untereinander abweichenden medienerzieherischen Einstellungen wird in der Folge sehr spannend sein. Gibt es neben der grossen Gruppe von Befragten, die zugleich eine frühe aktivhandlungsorientierte Medienbildung mit Medien ohne Bildschirm befürwortet, den Digitalmedieneinsatz jedoch erst viel später als sinnvoll erachtet, auch Gruppen, in welchen beispielsweise beides früh oder auch umgekehrt das Digitale früh und das Analoge spät als sinnvoll angesehen wird? Und welche Prädiktoren sagen die Zuordnung zu den Untergruppen am besten voraus: Das Alter der Befragten oder das ihrer Kinder, die eigene technische Versiertheit, die Verbundenheit mit spezifischen reformpädagogischen Ansätzen, usw.? 


\section{2 Überraschend hohe globale Elternzufriedenheit, mit Ausnahmen}

Im Licht der oben berichteten Studienlage, in der das Thema Bildung im digitalen Zeitalter als grösste «Baustelle» reformpädagogischer Bildungseinrichtungen aus Eltern- und Ehemaligensicht berichtet wird, überrascht, dass bei der globalen Abfrage der Elternzufriedenheit zwei Bereiche mit fast $90 \%$ sehr gut abschneiden. Zum Teil könnte diese sehr hohe Zufriedenheit auch auf die Tatsache zurückzuführen sein, dass Eltern an Waldorfschulen und an Montessori-Schulen (zumindest derjenigen in privater Trägerschaft) sich gezielt für das gewählte pädagogische Konzept entschieden haben und daher die hohe Zufriedenheit mit dem angenommenen guten Passungsverhältnis in Zusammenhang steht. Lediglich in den oberen Klassen (ca. ab Klasse 7) nimmt die Zufriedenheit betreffend unterrichtlichem Digitalmedieneinsatz ab. Oberstufen-Eltern (Klasse 10-13) sind etwa zur Hälfte unzufrieden darüber, dass dieser nicht, bzw. nicht oft und/oder gut genug umgesetzt wird. In den unteren Klassen dagegen ist die überwiegende Antwort, digitale Medien würden "genau richtig» eingesetzt. "Genau richtig» kann prinzipiell eine Zufriedenheit mit dem Einsatz oder dem Nicht-Einsatz der betreffenden Medien bedeuten. Die Ergebnisdeutung wird erst möglich durch den Abgleich mit den Angaben darüber, was die Eltern für die betreffende Altersstufe als sinnvoll erachten. Der Bereich «Produzieren und Präsentieren» illustriert exemplarisch, wie auf eine hohe Eltern-Zufriedenheit mit dem NichtEinsatz digitaler Medien in den unteren Klassen geschlossen werden kann.

Als sehr gewinnbringend hat sich die Verwendung eines in weiten Teilen für alle drei Gruppen übereinstimmenden, innovativen Befragungsinstrumentes erwiesen. Eine Methodik also, die auf Vergleichbarkeit abzielt und durch weitere Analysen die Untersuchung von Unterschieden und Gemeinsamkeiten zwischen den drei Gruppen ermöglicht, was auch ausserhalb des reformpädagogischen Kontextes einen hohen Erkenntnisgewinn versprechen lässt.

\subsection{Ist die Dichotomie von «bewahrpädagogischer» vs. «handlungsorientierter» Einstellung haltbar?}

Gängige Definitionen und Kritiken einer «bewahrpädagogischen» Erziehungshaltung im Diskurs um Bildung im digitalen Zeitalter werden durch die Befragungsergebnisse zum Teil bestätigt und zum Teil grundsätzlich in Frage gestellt. So bestätigt sich die bisher empirisch nicht breit gestützte Annahme, dass Eltern an reformpädagogischen Schulen einen frühen Digitalmedieneinsatz im Unterricht nicht befürworten, dass sie also, vereinfacht ausgedrückt, mehrheitlich «Bewahrpädagoginnen und -pädagogen» seien. Die Kritik jedoch, diesen Ansatz gelte es zu überwinden, da solche Positionen von defizitorientierten Annahmen über die Kind-Erwachsenen-Differenz ausgingen, mangelnde Kompetenzen und die Schutzbedürftigkeit von Kindern hervorhöben und damit die Befähigung vernachlässigten (Kammerl et al. 2020, 26), 
erweist sich u. E. als eher unzutreffend. Eine Förderung von Medienkompetenz durch die Bevorzugung analoger Medien in Kombination mit der Stärkung allgemeiner Lebenskompetenzen und einer Beschränkung der Verfügbarkeit digitaler Medien wird im medienpädagogischen Fachdiskurs immer noch überwiegend auf eine technische Unversiertheit sowie auf eine medienskeptische Haltung zurückgeführt (PfaffRüdiger, Brüggen, und Schubert 2020, 93ff).

Die in der MünDig-Studie aufgefundene, sehr verbreitete Befürwortung einer aktiv-handlungsorientierten Medienpädagogik ab Klasse 1 in allen 6 Bereichen des Medienkompetenzrahmens NRW, erscheint alles andere als «defizitorientiert», auch die abgefragten technischen Fähigkeiten der Befragten liegen im oberen Mittelfeld. Theoretisch ergiebig könnte hier in Zukunft insbesondere eine Korrelationsanalyse sein: Halten Eltern mit hohen eigenen technischen Fähigkeiten einen Einsatz digitaler Medien eher früher oder später für sinnvoll als weniger technisch versierte Eltern? Oder gibt es beide Trends in zwei Subgruppen? Die Handlungsorientierung sollte nach Auffassung der befragten Eltern jedenfalls zunächst durch den Einsatz von Medien ohne Bildschirm, später dann ergänzend durch Einsatz digitaler Bildschirmmedien erfolgen. Ist das noch Bewahrpädagogik oder schon aktiv-handlungsorientierte Medienpädagogik? Die in der Literatur vielfach angenommene Dichotomie, man könne nur eine der beiden Positionen vertreten, erweist sich u. E. für die befragten Eltern als nicht haltbar: Die Eltern möchten ihre Kinder sowohl durch zur jeweiligen Entwicklungsphase passende Medien in der Schule befähigen, und sie zugleich vor Digital-Risiken schützen, sowohl durch Stärkung ihrer realweltlichen sozialen und individuellen Ressourcen wie auch durch Zugangsbegrenzungen (vgl. Abschnitt 5.1.2). Dies stellt, am Rande bemerkt, auch die Einteilung von familiären Medienerziehungsstilen in entweder «active» oder «restrictive mediation» in Frage (Mößle und Bleckmann 2015). Die beschriebene Gruppe von Eltern grenzt sich sehr deutlich ab von der Annahme, die Rolle von Eltern als «zuständige Regulatoren der kindlichen Mediennutzung und Medienausstattung» (Moser 2019, 61) verliere stark an Bedeutung. Dies erweist sich erstens als mit dem Kindheitskonstrukt der Befragten eindeutig nicht kompatibel, zweitens aber auch nicht mit Forschungsbefunden zu wirksamen Strategien der Prävention von Digital-Risiken (Bleckmann und Mößle 2014). Für die Mehrzahl der Befragten, wie auch für die in der Einleitung genannten IT-Experten, die ihre Kinder im Silicon Valley auf eine reformpädagogische Schule schicken, müsste die Kritik im Lichte der vorgelegten Forschungsergebnisse relativiert oder zumindest neuformuliert werden. Insofern könnte dieser Beitrag einen Impuls setzen für das Ernstnehmen von Eltern als Mitgestalter (medien-)pädagogischer Prozesse auf Augenhöhe mit pädagogischen Fachkräften, wie auch für die Anschlussfähigkeit der «reformpädagogisch orientierten Medienpädagogik» an den medienpädagogischen Fachdiskurs. 


\section{Literatur}

Antonovsky, Aaron. 1997. Salutogenese: Zur Entmystifizierung der Gesundheit. Edited by Alexa Franke. Forum für Verhaltenstherapie und psychosoziale Praxis Band 36. Tübingen: dgvt Verlag.

Baacke, Dieter. 1997. Medienpädagogik. Grundlagen der Medienkommunikation;. 6. Aufl. Tübingen: Niemeyer.

Bandilla, Wolfgang. 2015. Online-Befragungen. Mannheim: SDM-Survey Guidelines (GESIS Leibniz Institute for the Social Sciences). https://doi.org/10.15465/gesis-sg_003.

Barz, Heiner, Hrsg. 2019. Bildung und Schule - Elternstudie 2019: Einstellungen von Eltern in Deutschland zur Schulpolitik. Münster: Waxmann.

Bitzer, Eva M., Paula Bleckmann, und Thomas Mößle. 2014. «Prävention problematischer und suchtartiger Bildschirmmediennutzung. Eine deutschlandweite Befragung von Praxiseinrichtungen und Experten». KFN-Forschungsbericht 125. Unveröffentlichtes Manuskript. https://kfn.de/wp-content/uploads/Forschungsberichte/FB_125.pdf.

Bleckmann, Paula. 2020. Medienmündig: Wie unsere Kinder selbstbestimmt mit dem Bildschirm umgehen lernen. 7. Aufl. Stuttgart: Klett-Cotta.

Bleckmann, Paula, Elisabeth Denzl, und Benjamin Streit. 2021. «Medienmündig werden: Konzeptionelle und empirische Annäherungen an ein erweitertes Verständnis von Medienbildung jenseits vom Einsatz von Tablets in Kitas». Frühe Kindheit, im Erscheinen.

Bleckmann, Paula, und Thomas Mößle. 2014. «Position zu Problemdimensionen und Präventionsstrategien der Bildschirmnutzung». SUCHT60 (4): 235-47. https://doi.org/10.1024/09395911.a000313.

Bleckmann, Paula, und Valentina Nartschenko. 2019. "Kampf um die Medienhoheit im Kinderzimmer - Schule als Teil der Lösung oder als Teil des Problems?». In Bildung und Schule Elternstudie 2019: Einstellungen von Eltern in Deutschland zur Schulpolitik, herausgegeben von Heiner Barz, 83-94. Münster: Waxmann.

Bleckmann, Paula, und Brigitte Pemberger. 2021. «Bildung und Digitalisierung - Technikfolgenabschätzung und die Entzauberung „digitaler Bildung“ in Theorie und Praxis». In Wie wir leben wollen. Kompendium zu Technikfolgen von Digitalisierung, Vernetzung und Künstlicher Intelligenz, herausgegeben von Frank Schmiedchen, Klaus P. Kratzer, Jasmin S. Link, und Heinz Stapf-Finé, 191-210. Berlin: Logos Verlag. https://doi.org/10.30819/5363. https://doi.org/10.30819/5363.

Bleckmann, Paula, Frederik Tetzlaff, Brigitte Pemberger, Benjamin Streit, und Julia Kernbach. 2021a im Erscheinen. MünDig-Studie Montessori: Praxis und Einstellungen zur Medienerziehung von PädagogInnen und Eltern an Kinderhäusern und Schulen.

Bleckmann, Paula, Frederik Tetzlaff, Brigitte Pemberger, Benjamin Streit, und Julia Kernbach. 2021b im Erscheinen. MünDig-Studie Waldorf: Praxis und Einstellungen zur Medienerziehung von PädagogInnen und Eltern an Krippen, Kindergärten und Schulen.

Bleckmann, Paula, und Jasmin Zimmer. 2016. «Media education habitus and practice of teachers - how can we understand and change it». EAPRIL, Portugal, 22. November. 
Böcking, S. 2006. «Elterlicher Umgang mit kindlicher Fernsehnutzung. Test einer deutschsprachigen Skala und erste Befunde für die Deutschschweiz.». Medien \& Kommunikationswissenschaft 54: 599-619.

Brinda, Torsten, Niels Brüggen, Ira Diethelm, Thomas Knaus, Sven Kommer, Christine Kopf, Petra Missomelius, Rainer Leschke, Friederike Tilemann, und Andreas Weich. 2019. «Frankfurt-Dreieck zur Bildung in der digital vernetzten Welt». https://dagstuhl.gi.de/frankfurtdreieck.de. Zugriff am 5. Januar 2021.

Brodbeck, Heinz. 2020. «Entwicklungsfelder für die Rudolf Steiner Schulen (Triangulationsstudie)». https://www.academia.edu/44310601/Entwicklungsfelder_f \%C3\%BCr_Waldorfschulen_und_Rudolf_Steiner_Schulen_Triangulationsstudie_.

Couper, Mick. 2008. Designing Effective Web Surveys. Cambridge, New York: Cambridge University Press.

Dietz, Karl Martin. 2011. "Gemeinsam in die Zukunft: Zur Erziehungspartnerschaft von Eltern und Lehrern im Rahmen der Selbstverwaltung». In Waldorfschule heute: Eine Einführung, herausgegeben von Peter Loebell, und Karl M. Dietz. 1. Aufl. der Neuausg, 283-318. Stuttgart: Verl. Freies Geistesleben.

Feierabend, Sabine, Theresa Plankenhorn, und Thomas Rathgeb. 2019. «KIM-Studie 2018 Kindheit, Internet, Medien: Basisuntersuchung zum Medienumgang 6- bis 13-Jähriger in Deutschland». Unveröffentlichtes Manuskript, zuletzt geprüft am 8. Januar 2021. https:// www.mpfs.de/studien/kim-studie/2018/.

Frielingsdorf, Volker. 2019. Geschichte der Waldorfpädagogik: Von ihrem Ursprung bis zur Gegenwart. Weinheim: Julius Beltz.

Graudenz, Ines. 2021. "Herausforderungen für die Waldorfschule in der Zukunft». In Wir waren auf der Waldorfschule: Ehemalige als Experten in eigener Sache, herausgegeben von Dirk Randoll, und Jürgen Peters, 94-102. Weinheim: Beltz Juventa.

Grobbin, Alexander. 2016. «Digitale Medien: Beratungs-, Handlungs- und Regulierungsbedarf aus Elternperspektive». Unveröffentlichtes Manuskript, zuletzt geprüft am 6. Januar 2021. https://www.dji.de/en/media/literature-search/detailansicht/literatur/23188-digitale-medien-beratungs-handlungs-und-regulierungsbedarf-aus-elternperspektive.html.

Hauser, Urs, Juraj Hromkovič, Petra Klingenstein, Regula Lacher, Pascal Lütscher, und Jacqueline Staub. 2020. Einfach Informatik Zyklus 1. 1. Auflage.

Herbst, Theresia, und Dieter Bergmayr. 2020. «DigitaleMedieninderVorbereitetenUmgebung». In Montessori-Pädagogik: Das Kind im Mittelpunkt, herausgegeben von Franz Hammerer, Theresia Herbst, und Wilhelm Weinhäupl, 258-78. Braunschweig: Westermann.

Hromkovič, Juraj, und Regula Lacher. 2019. Einfach Informatik 5/6: Primarstufe. 1. Auflage.

Hübner, Edwin. 2020. «Medien und Schule. Neun Thesen zu einer entwicklungsorientierten Medienpädagogik». In Studienbuch Waldorf-Schulpädagogik, herausgegeben von Angelika Wiehl, 247-62. UTB 5231. Bad Heilbrunn: Klinkhardt.

Hüther, Jürgen, und Bernd Schorb, Hrsg. 2005. Grundbegriffe Medienpädagogik. München: kopaed. 
Irion, Thomas. 2020. «Digitale Bildung in der Grundschule: Grundlegende Bildung in der digital geprägten und gestaltbaren, mediatisierten Welt». In Digitale Bildung im Grundschulalter: Grundsatzfragen zum Primat des Pädagogischen, herausgegeben von Mareike Thumel, Rudolf Kammerl, und Thomas Irion, 49-81. München: kopaed.

Kammerl, Rudolf, Andreas Dertinger, Melanie Stephan, und Mareike Thumel. 2020. «Digitale Kompetenzen und Digitale Bildung als Referenzpunkte für Kindheitskonstruktion im Mediatisierungsprozess». In Digitale Bildung im Grundschulalter: Grundsatzfragen zum Primat des Pädagogischen, herausgegeben von Mareike Thumel, Rudolf Kammerl, und Thomas Irion. München: kopaed.

Kernbach, Julia, und Benjamin Streit. 2021. «Wollen sie am Ende dasselbe? Was sich Erzieher*innen und Eltern für Medienbildung in reformpädagogischen Kindergärten wünschen. Erste Ergebnisse anhand der MünDig-Stdie». Symposium auf der PdFK Konferenz für Nachwuchwissenschaftler:innen, Vechta, 24. September.

Kullack-Ublick, Henning, und Arbeitskreis Medienmündigkeit und Waldorfpädagogik im Bund der Freien Waldorfschulen, Hrsg. 2015. Struwwelpeter 2.0. Zugriff am 15. Januar 2021. https://www.waldorfschule.de/fileadmin/downloads/Blickpunkte_Reader/Medienbroschuere_Struwwelpeter_2.0.pdf.

Kultusministerkonferenz, Hrsg. 2016. Bildung in der digitalen Welt. Zugriff am 6. Januar 2021. https://www.kmk.org/themen/bildung-in-der-digitalen-welt/strategie-bildung-in-der-digitalen-welt.html.

Lee, Kerry. 2003. «So What Do Parents Want and Expect from a Technology Education Programme? - An Exploration». International Journal of Technology and Design Education 13 (2): 105-15. https://doi.org/10.1023/A:1024186332434.

Livingstone, Sonia, Alicia Blum-Ross, und Zhang Dongmiao. 2018. What do parents think and do, about their children 's online privacy? Parenting for a digital future: Survey report 3. London/UK: Department of Media and Communications, The London School of Economics and Political Scene. http://eprints.lse.ac.uk/id/eprint/87954.

Lorenz, Ramona, Julia Gerick, Renate Schulz-Zander, und Birgit Eickelmann. 2014. «Computerund informationsbezogene Kompetenzen von Mädchen und Jungen im internationalen Vergleich». In ICILS 2013. Computer- und informationsbezogene Kompetenzen von Schülerinnen und Schülern in der 8. Jahrgangsstufe im internationalen Vergleich, herausgegeben von Wilfried Bos, Birgit Eickelmann, Julia Gerick, Frank Goldhammer, Heike Schaumburg, Knut Schwippert, Martin Senkbeil, Renate Schulz-Zander, und Heike Wendt, 231-63. Münster: Waxmann Verlag. https://doi.org/10.25656/01:11459.

LVR Zentrum für Medien und Bildung. «Medienkompetenzrahmen NRW». https://medienkompetenzrahmen.nrw.

Marotzki, Winfried, und Benjamin Jörissen. 2008. «Medienbildung». In Handbuch Medienpädagogik, herausgegeben von Uwe Sander, Friederike von Gross, und Kai-Uwe Hugger, 100-109. Wiesbaden: VS Verlag für Sozialwissenschaften. https://doi.org/10.1007/978-3531-91158-8_11. 
Moser, Heinz. 2019. Einführung in die Medienpädagogik: Aufwachsen im digitalen Zeitalter. 6., überarbeitete und aktualisierte Auflage 2019. Wiesbaden: Springer Fachmedien Wiesbaden GmbH. https://doi.org/10.1007/978-3-658-23208-5.

Mößle, Thomas, und Paula Bleckmann. 2015. «Talk or act? Effects of screen availability vs. effects of parental mediation style on children`s problematic use». „Changing behaviour without talking: automatic processes and the regulation of behaviour", Ljublijana, EUSPR Coference, 23. November.

Nathanson, A. I. 1999. «Identifying and Explaining the Relationship Between Parental Mediation and Children's Aggression». Communication Research 26 (2): 124-43.

Onken, Annette. 2017. «Montessori-Erziehung in Familie und Spielgruppe». In Grundgedanken der Montessori-Pädagogik: Quellentexte und Praxisberichte, herausgegeben von Harald Ludwig, Paul Oswald, und Günter Schulz-Benesch. Freiburg, Basel, Wien: Herder.

Peitz, Julia, Marius Harring, und Stephan D. Müller. 2017. «Eltern in reformpädagogischen Settings». In Handbuch Reformpädagogik, herausgegeben von Till-Sebastian Idel, und Heiner Ullrich, 324-37. Beltz Handbuch. Weinheim, Basel: Beltz Verlagsgruppe.

Penert, Katinka. 2019. Informatik in Bewegung: Computer Science unplugged. München: GRIN Verlag. https://www.grin.com/document/490036.

Penert, Katinka, und Brigitte Pemberger. 2020. «Medienerziehung in der Waldorf-Kindheitspädagogik». In Studienbuch Waldorf-Schulpädagogik, herausgegeben von Angelika Wiehl, 190-204. UTB 5231. Bad Heilbrunn: Klinkhardt.

Pfaff-Rüdiger, Senta, Niels Brüggen, und Gisela Schubert. 2020. «Wir können uns davor überhaupt nicht verschließen: Medienpädagogische Kompetenz von Fachkräften in Kindergärten und Horten für eine „Digitale Bildung“». In Digitale Bildung im Grundschulalter: Grundsatzfragen zum Primat des Pädagogischen, herausgegeben von Mareike Thumel, Rudolf Kammerl, und Thomas Irion. München: kopaed.

Pörksen, Bernhard. 2018. Die große Gereiztheit: Wege aus der kollektiven Erregung. 1. Auflage. München: Carl Hanser Verlag GmbH \& Co. KG. https://doi.org/10.3139/9783446259560.

Redecker, Christine. 2017. European Framework for the Digital Competence of Educators: DigCompEdu. Brüssel: Europäische Kommission. https://doi.org/10.2760/178382. https://doi. org/10.2760/178382.

Richter, Tobias, Hrsg. 2006/2016. Pädagogischer Auftrag und Unterrichtsziele - vom Lehrplan der Waldorfschule. 4. Aufl. Stuttgart: Verlag Freies Geistesleben.

Schmidt, Robin. 2020. «ICT-Professionalisierung und ICT-Beliefs. Professionalisierung angehender Lehrpersonen in der digitalen Transformation und ihre berufsbezogenen Überzeugungen über digitale Informations- und Kommunikationstechnologien (ICT)». Dissertation, Universität Basel. https://doi.org/10.5451/unibas-007196575.

Schwill, Andreas. 1993. «Fundamentale Ideen der Informatik». ZDM - Zentralblatt für Didaktik der Mathematik 20-31 (25).

Skiera, Ehrenhard. 2010. Reformpädagogik in Geschichte und Gegenwart: Eine kritische Einführung. München: OLDENBOURG WISSENSCHAFTSVERLAG. https://doi. org/10.1524/9783486851328. 
Stange, Waldemar. 2013. «Präventions- und Bildungsketten - Elternarbeit als Netzwerkaufgabe». In Erziehungs- und Bildungspartnerschaften: Praxisbuch zur Elternarbeit, herausgegeben von Waldemar Stange, 17-69. Wiesbaden: Springer VS. https://doi.org/10.1007/978-3531-94295-7_1.

Statistisches Bundesamt. 2019. «Pressemitteilung Nr. 364 vom 18. September 2019». https:// www.destatis.de/DE/Presse/Pressemitteilungen/2019/09/PD19_364_211.html.

Stein, Barbara, Hrsg. 2017. Die Montessori-Grundschule in Theorie und Praxis. Montessori-Praxis. Freiburg, Basel, Wien: Herder.

Sümmchen, Corinna. 2019. «Analoges soziales Netzwerk oder Social Media Unplugged: Handlungsorientierte Prävention von Cyber-Risiken». Erlebnis und Lernen (3\&4): 40-43.

Te Wildt, Bert. 2015. Digital Junkies. Internetabhängigkeit und ihre Folgen für uns und unsere Kinder. München: Droemer.

Tetzlaff, Frederik, und Paula Bleckmann. 2019. «Digitalisierung und Pädagogik - weit mehr als nur „Tablets im Unterricht“». In Bildung und Schule - Elternstudie 2019: Einstellungen von Eltern in Deutschland zur Schulpolitik, herausgegeben von Heiner Barz, 69-82. Münster: Waxmann.

Thom, Sabrina, Julia Behrens, Ulrich Schmid, und Lutz Goertz. 2018. «Monitor Digitale Bildung: Digitales Lernen an Grundschulen». https://doi.org/10.11586/2017040.

Valkenburg, Patti M., Marina Krcmar, Allerd L. Peeters, und Nies M. Marseille. 1999. "Developing a scale to assess three styles of television mediation: "Instructive mediation," "restrictive mediation," and "social coviewing" ». Journal of Broadcasting \& Electronic Media 43 (1): 52-66. https://doi.org/10.1080/08838159909364474.

Werner-Andrews, Sarah. 2017. «Eltern als Partner - Wie eine Kultur des Respekts und der Zusammenarbeit mit Eltern geschaffen werden kann». Montessori Zeitschrift der Deutschen Montessori Gesellschaft e.V. (61): 48-55.

Wiesmann, Bettina M. 2019. «Stellungnahme der Kinderkommission des Deutschen Bundestages zum Thema „Kindeswohl und digitalisierte Gesellschaft: Chancen wahrnehmen - Risiken bannen“: Kommissionsdrucksache 19. Wahlperiode 19/05». Unveröffentlichtes Manuskript, zuletzt geprüft am 6. Januar 2021. https://www.bundestag.de/resource/blob/6 51028/0de1b58a7b242fe62c293a19f00cb055/2019-07-10-Stellungnahme-Kindeswohl-und -digitalisierte-Gesellschaft-data.pdf.

Wolf, Karsten D. 2017. «Mediatisierung in reformpädagogischen Lernkulturen». In Handbuch Reformpädagogik, herausgegeben von Till-Sebastian Idel, und Heiner Ullrich, 338-52. Beltz Handbuch. Weinheim, Basel: Beltz Verlagsgruppe.

Zimmer, Jasmin, und Klaus Martin Zimmer. 2020. «Ich sehe was, was Du nicht siehst! DIAEDI: ein Handlungs-Modell für die Unterstützung der Verarbeitung von Medienerlebnissen». Bildungsforschung 18 (2 (Frühkindliche Bildung und Entwicklung)). https://doi.org/10.25539/ bildungsforschun.v2i18.301.

Wir bedanken uns bei Frederik Tetzlaff für die Erstellung des Erhebungsinstrumentes, die Betreuung der MünDig-Studie im Erhebungszeitraum und die Bereitstellung der Datensätze. 


\begin{tabular}{|c|c|c|c|c|c|c|c|c|c|c|c|c|c|c|c|}
\hline & & 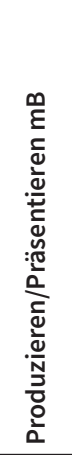 & 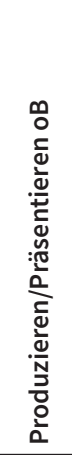 & 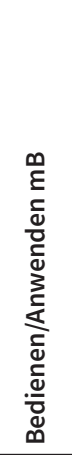 & 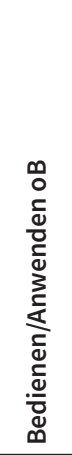 & 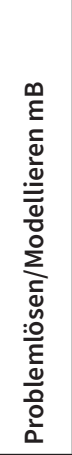 & 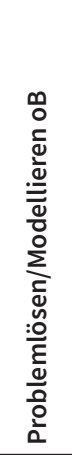 & 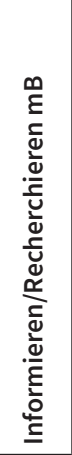 & 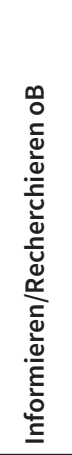 & 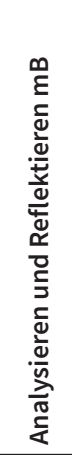 & 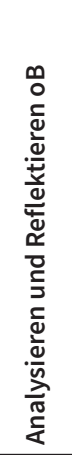 & 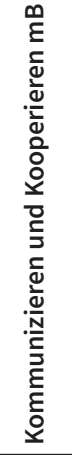 & 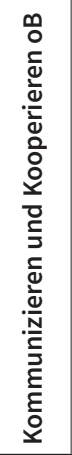 & 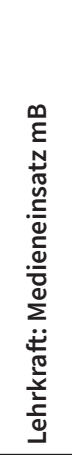 & 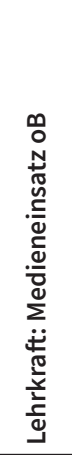 \\
\hline \multirow[t]{4}{*}{ Kl. 1-3 (N = 841 - 1049) } & genau richtig & 51,4 & 67,5 & 56,3 & 71,3 & 49,4 & 58,7 & 52,7 & 62 & 47,1 & 57 & 51,7 & 67,3 & 54,9 & 74 \\
\hline & zu selten & 10,3 & 8,3 & 10,9 & 8,3 & 11,5 & 14,4 & 11,8 & 16,2 & 12,8 & 14,3 & 9 & 9,2 & 10,7 & 6,1 \\
\hline & zu oft & 1,3 & 0,3 & 1,9 & 0,2 & 49,4 & 0,2 & 2,7 & 0,5 & 1,1 & 0,2 & 2,2 & 0,5 & 2,1 & 0,9 \\
\hline & weiss nicht & 37,1 & 23,9 & 30,9 & 20,3 & 38,2 & 26,6 & 32,7 & 21,3 & 39 & 28,5 & 37,1 & 23 & 32,2 & 18,9 \\
\hline \multirow[t]{4}{*}{ Kl. $4-6(N=448-573)$} & genau richtig & 56,5 & 70,9 & 60,9 & 71,4 & 49,5 & 58,3 & 56,6 & 59,3 & 47 & 58,3 & 52,7 & 69,4 & 54 & 74,2 \\
\hline & zu selten & 16,5 & 16,8 & 15,7 & 15,8 & 17,8 & 22,5 & 21 & 31,7 & 24,5 & 26,3 & 12,7 & 15,1 & 17,4 & 10,7 \\
\hline & zu oft & 1,2 & 0,7 & 3,7 & 0,9 & 1,6 & 0,2 & 4,1 & 0,2 & 1,5 & 0 & 5,4 & 0 & 3,1 & 0,7 \\
\hline & weiss nicht & 25,8 & 11,7 & 19,6 & 11,9 & 31,1 & 19 & 18,3 & 8,8 & 27 & 15,4 & 29,2 & 15,5 & 25,4 & 14,4 \\
\hline \multirow[t]{4}{*}{ Kl. 7-9 (N = $372-430)$} & genau richtig & 51,4 & 71,9 & 43,6 & 60,1 & 35,6 & 51,1 & 46,2 & 49,9 & 34,5 & 45,2 & 44,5 & 61,1 & 46,8 & 67,9 \\
\hline & zu selten & 31,1 & 20,7 & 30,7 & 25,1 & 32,1 & 28,8 & 31 & 40,2 & 40,8 & 38,4 & 26,9 & 27,2 & 26,9 & 17,6 \\
\hline & zu oft & 1,6 & 1,6 & 7 & 1,7 & 3 & 0,5 & 8,4 & 1 & 3,1 & 0 & 8,3 & 0,5 & 3,8 & 1,6 \\
\hline & weiss nicht & 15,9 & 5,8 & 18,7 & 13,1 & 29,3 & 19,6 & 14,5 & 8,9 & 21,6 & 16,4 & 20,3 & 11,1 & 22,6 & 12,8 \\
\hline \multirow[t]{4}{*}{ Kl. $10-13(N=214-258)$} & genau richtig & 42,2 & 64 & 35,9 & 55,5 & 24,6 & 46,1 & 39,1 & 47,2 & 28,9 & 47,4 & 33,6 & 52,7 & 40,7 & 63,6 \\
\hline & zu selten & 39,1 & 24 & 37,5 & 28,7 & 36,7 & 27 & 37,3 & 43,3 & 47,4 & 39,5 & 31,4 & 29,7 & 32,7 & 18,2 \\
\hline & zu oft & 2,7 & 1,2 & 10,1 & 1,2 & 4,2 & 0,8 & 13,7 & 0,4 & 3,9 & 0 & 12,7 & 1,8 & 2,8 & 0,5 \\
\hline & weiss nicht & 15,9 & 10,9 & 16,5 & 14,6 & 34,6 & 26,1 & 9,9 & 9 & 19,7 & 13,2 & 22,3 & 15,8 & 23,8 & 17,8 \\
\hline \multicolumn{2}{|l|}{$\mathrm{N}$ (gesamt) } & 2290 & 2310 & 2217 & 2229 & 2088 & 2116 & 2032 & 2051 & 1957 & 1974 & 1915 & 1936 & 1875 & 1897 \\
\hline
\end{tabular}

Tab. 1.: Elternzufriedenheit hinsichtlich der ersten sieben abgefragten Bildungsziele in \% (siehe Abb. 4). 


\begin{tabular}{|c|c|c|c|c|c|c|c|}
\hline & & 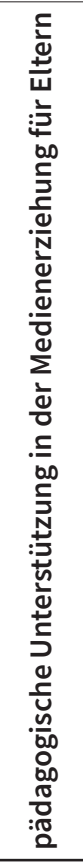 & 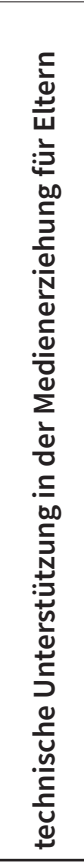 & 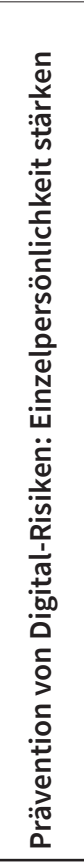 & 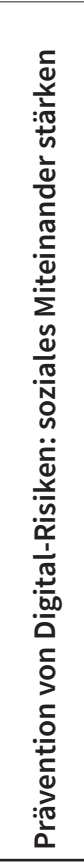 & 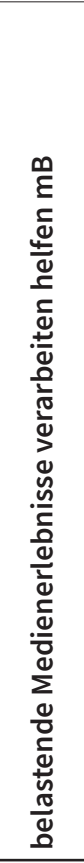 & 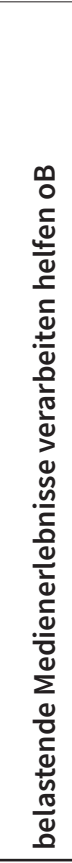 \\
\hline \multirow[t]{4}{*}{ Kl. $1-3(N=841$ - 1049) } & genau richtig & 40,4 & 23,8 & 72,5 & 76,6 & 30,9 & 33,6 \\
\hline & zu selten & 43 & 45,3 & 18,7 & 15,4 & 15,4 & 17,8 \\
\hline & zu oft & 1,5 & 0,5 & 0,5 & 0,1 & 0,7 & 0,4 \\
\hline & weiss nicht & 15,1 & 30,4 & 8,2 & 7,8 & 53,1 & 48,2 \\
\hline \multirow[t]{4}{*}{ Kl. 4-6 (N = 448 - 573) } & genau richtig & 35,8 & 21,9 & 66,8 & 73,1 & 30,2 & 34,9 \\
\hline & zu selten & 56 & 58,8 & 26,4 & 21,5 & 25,4 & 30,2 \\
\hline & zu oft & 1,4 & 0,7 & 0,2 & 0,5 & 1,2 & 0 \\
\hline & weiss nicht & 6,7 & 18,6 & 6,5 & 4,8 & 43,1 & 34,9 \\
\hline \multirow[t]{4}{*}{ Kl. $7-9(N=372-430)$} & genau richtig & 27,9 & 14,8 & 63 & 72,2 & 23,8 & 29,1 \\
\hline & zu selten & 63,8 & 71,6 & 32,2 & 24 & 36,1 & 39 \\
\hline & zu oft & 2,4 & 0 & 0 & 0 & 0 & 0 \\
\hline & weiss nicht & 5,9 & 13,6 & 4,7 & 3,8 & 40,1 & 31,8 \\
\hline \multirow[t]{4}{*}{ Kl. $10-13(N=214-258)$} & genau richtig & 28,8 & 16,6 & 64,8 & 66,7 & 22,1 & 26,8 \\
\hline & zu selten & 58,6 & 63,3 & 29,1 & 28,2 & 38,5 & 38,1 \\
\hline & zu oft & 6,1 & 3,5 & 0,5 & 0 & 0,5 & 0 \\
\hline & weiss nicht & 6,6 & 16,6 & 5,6 & 5,1 & 39 & 35,1 \\
\hline N (gesamt) & & 1735 & 1735 & 1726 & 1724 & 1696 & 1703 \\
\hline
\end{tabular}

Tab. 2.: Elternbewertung der medienerzieherischen Tätigkeiten Bereiche 8-10 (siehe Abb. 6). 